\title{
О ПОЛИЭДРАЛЬНОМ ОЦЕНИВАНИИ МНОЖЕСТВ ДОСТИЖИМОСТИ В "РАСШИРЕННОМ" ПРОСТРАНСТВЕ ДЛЯ МНОГОШАГОВЫХ СИСТЕМ С НЕОПРЕДЕЛЕННЫМИ МАТРИЦАМИ И ИНТЕГРАЛЬНЫМИ ОГРАНИЧЕНИЯМИ
}

\author{
Е. К. Костоусова
}

\begin{abstract}
Рассматриваются задачи достижимости и построения оценок множеств достижимости (МД) многошаговых систем с исходно линейной структурой и неопределенностями в начальных условиях, матрицах и аддитивных воздействиях. Неопределенности стеснены заданными параллелепипедозначными, интервальными и интегральными неквадратичными ограничениями соответственно. Ввиду неопределенности в матрицах системы оказываются билинейного типа. МД рассматриваются не только в исходном пространстве $\mathbb{R}^{n}$, но и в "расширенном" пространстве $\mathbb{R}^{n+1}$, где последняя координата $\mu$ соответствует текущему резерву аддитивного входного воздействия. Дано точное описание МД $\mathcal{Z}[k]$ в "расширенном" пространстве с помощью многозначных рекуррентных соотношений. При этом используется представление множеств в виде объединения их $\mu$-сечений, а рекуррентные соотношения включают операции с множествами, одна из которых (умножение на интервальную матрицу) действует на каждое сечение независимо, а еще одна комбинирует операции суммы Минковского и объединения по сечениям. МД $\mathcal{X}[k]$ в $\mathbb{R}^{n}$ определяются сечениями $\mathcal{Z}[k]$, соответствующими $\mu=0$. Однако вычислить точно $\mathcal{Z}[k]$ из вышеупомянутых соотношений обычно трудно. Предлагаются способы построения параметризованных семейств внешних и внутренних полиэдральных оценок множеств $\mathcal{Z}[k]$ в виде политопов специального типа. На их основе строятся внешние параллелепипедозначные и внутренние параллелотопозначные оценки для $\mathcal{X}[k]$. Все оценки находятся по явным формулам из систем рекуррентных соотношений.
\end{abstract}

Ключевые слова: множество достижимости, интегральные ограничения, неопределенность в матрице, полиэдральные оценки, параллелепипеды, параллелотопы.

E. K. Kostousova. On polyhedral estimation of reachable sets in the "extended" space for discrete-time systems with uncertain matrices and integral constraints.

The problems of reachability and construction of estimates of reachable sets are considered for discrete-time systems with initially linear structure and uncertainties in the initial conditions, matrices, and additive input actions. The uncertainties are restricted by given parallelepiped-valued, interval, and integral nonquadratic constraints, respectively. The systems under consideration turn out to be of bilinear type due to the uncertainty in the matrices. The reachable sets are considered not only in the original space $\mathbb{R}^{n}$ but also in the "extended" space $\mathbb{R}^{n+1}$, where the last coordinate $\mu$ corresponds to the current reserve of the additive input action. An exact description is given for the reachable sets $\mathcal{Z}[k]$ in the "extended" space using multivalued recurrence relations. Here, the representation of sets in the form of the union of their $\mu$-sections is used, and the recurrence relations include operations with sets; one of the operations (multiplication by an interval matrix) acts on each cross-section independently, and another combines the Minkowski sum and the union over cross-sections. The reachable sets $\mathcal{X}[k]$ in $\mathbb{R}^{n}$ are determined by the cross-sections of $\mathcal{Z}[k]$ corresponding to $\mu=0$. However, it is usually difficult to calculate $\mathcal{Z}[k]$ exactly from the above relations. Methods are proposed for the construction of parametrized families of external and internal polyhedral estimates of the sets $\mathcal{Z}[k]$ in the form of polytopes of a special type. On this basis, external parallelepiped-valued and internal parallelotope-valued estimates of $\mathcal{X}[k]$ are constructed. All estimates are found by explicit formulas from systems of recurrence relations.

Keywords: reachable set, integral constraints, uncertain matrix, polyhedral estimates, parallelepipeds, parallelotopes.

MSC: 93B03, 93C10, 93C55, 93C41, 93B40

DOI: $10.21538 / 0134-4889-2020-26-1-141-155$

\section{Введение}

Решение многих задач управления и оценивания в условиях неопределенности основывается на построении множеств достижимости (МД), их аналогов и трубок траекторий, описывающих динамику этих множеств [1-4]. Поскольку их точное построение является, как правило, 
непростой задачей, активно развиваются численные методы, в том числе полиэдральные [5] и пиксельные [6], а также значительно менее трудоемкие методы, основанные на аппроксимации множеств простыми областями, в частности, эллипсоидами [3;7-9], зонотопами [10], параллелотопами (параллелепипедами) [11-13] и боксами (интервальными векторами) [14] (здесь для примера были упомянуты лишь некоторые из многочисленных публикаций; см. также ссылки в них).

Работа посвящена построению и оцениванию МД многошаговых систем с исходно линейной структурой и неопределенностями в начальных условиях, матрицах и аддитивных воздействиях, причем последние стеснены интегральными ограничениями. Подход, предложенный в [13] для линейных систем, развивается здесь на более сложный случай систем с билинейностью, вызванной неопределенностью в матрицах, которая может приводить к возможной невыпуклости МД (см., например, [15-17]) и тем самым привносит в исследование дополнительные трудности. При этом для построения оценок привлекаются также конструкции из [18]. Дано подробное описание и обоснование соотношений, доставляющих точные представления МД, и способов построения параметризованных семейств внешних и внутренних полиэдральных оценок для них. При этом рассматриваются МД $\mathcal{X}[k]$ в исходном пространстве $\mathbb{R}^{n}$ и МД $\mathcal{Z}[k]$ в "расширенном" пространстве $\mathbb{R}^{n+1}$, где последняя координата $\mu$ соответствует текущему резерву аддитивного входного воздействия; указывается явная связь между этими множествами. Для множеств $\mathcal{Z}[k]$ строятся оценки в виде политопов специального типа. Определенные сечения таких оценок дают внешние параллелепипедозначные и внутренние параллелотопозначные оценки для $\mathcal{X}[k]$. Упомянем, что ранее в [19] были анонсированы алгоритмы построения внешних оценок и приведен пример их нахождения.

Исследуемые системы можно рассматривать как дискретные аналоги импульсных систем. В этом плане полезно отметить публикации, в которых исследованы свойства и аппроксимации МД дифференциальных систем с интегральными ограничениями на управления из $L_{p}$ с $p>1[6 ; 9]$ и для импульсных систем [4], для систем с геометрическими ограничениями и билинейностью $[16 ; 17 ; 20]$ и для комбинации импульсных систем с билинейностью [15].

В работе используются следующие обозначения: $\mathbb{R}^{n}$ и $\mathbb{R}^{n \times m}$ - линейные пространства вещественных $n$-векторов и $n \times m$-матриц соответственно; $\top-$ знак транспонирования; $(x, y)=x^{\top} y$ - скалярное произведение для $x, y \in \mathbb{R}^{n} ;\|x\|_{2}=\left(x^{\top} x\right)^{1 / 2}$ и $\|x\|_{\infty}=\max _{1 \leq i \leq n}\left|x_{i}\right|$ разные нормы вектора $x=\left(x_{1}, \ldots, x_{n}\right)^{\top} \in \mathbb{R}^{n} ; \mathrm{e}^{i}=(0, \ldots, 0,1,0, \ldots, 0)^{\top} \in \mathbb{R}^{n}$ - единичный орт вдоль оси $0 x_{i}$ (единица стоит на $i$-м месте); е $=(1,1, \ldots, 1)^{\top} ; A=\left\{a_{i}^{j}\right\}=\left\{a^{j}\right\}$ - матрица с элементами $a_{i}^{j}$ и со столбцами $a^{j}$ (верхним индексом нумеруются столбцы, нижним компоненты векторов); 0 - нулевая матрица (вектор) произвольной размерности; $I$ - единичная матрица; $\operatorname{Abs} A-$ матрица абсолютных величин элементов матрицы $A=\left\{a_{i}^{j}\right\} \in \mathbb{R}^{n \times m}$ : $\operatorname{Abs} A=\left\{\left|a_{i}^{j}\right|\right\} ; \operatorname{diag} \pi, \operatorname{diag}\left\{\pi_{i}\right\}$ - диагональная матрица с компонентами $\pi_{i}$ вектора $\pi$ на диагонали; $\operatorname{det} A-$ определитель матрицы $A ;\|A\|=\max _{1 \leq i \leq n} \sum_{j=1}^{m}\left|a_{i}^{j}\right|-$ норма матрицы $A \in \mathbb{R}^{n \times m}$, индуцированная нормой $\|x\|_{\infty} ;$ со $\mathcal{Q}$ - выпуклая оболочка множества $\mathcal{Q} \subset \mathbb{R}^{n} ; \partial \mathcal{Q}-$ граница множества $\mathcal{Q} ; \rho(l \mid \mathcal{Q})=\sup \left\{l^{\top} x \mid x \in \mathcal{Q}\right\}-$ опорная функция множества $\mathcal{Q}$. Кроме того, используем для краткости обозначения типа $k=1, \ldots, n$ вместо $k=1,2, \ldots, n$.

\section{1. Постановка задачи}

Рассматривается многошаговая система

$$
\begin{gathered}
x[j]=A[j] x[j-1]+B[j] u[j]+v[j], \quad j=1, \ldots, N ; \\
x[0] \in \mathcal{X}_{0} \subset \mathbb{R}^{n} ; \quad \sum_{j=1}^{N}\|u[j]\|_{\infty} \leq \mu_{0} ; \\
u[j] \in \mathcal{K}[j] \subseteq \mathbb{R}^{n_{u}}, \quad j=1, \ldots, N .
\end{gathered}
$$


Здесь $x[j] \in \mathbb{R}^{n}$ - вектор состояния, $v[j] \in \mathbb{R}^{n}-$ известные воздействия, $B[j] \in \mathbb{R}^{n \times n_{u}}$ $\left(n_{u} \leq n\right)$. Начальное состояние $x[0]$ и входные воздействия $u[j] \in \mathbb{R}^{n_{u}}$ неизвестны, но стеснены ограничениями $(1.2),(1.3)$, где $\mathcal{X}_{0}$ - заданное выпуклое компактное множество, $\mu_{0}>0,\|u\|_{\infty}=$ $\max _{1 \leq i \leq n_{u}}\left|u_{i}\right|, \mathcal{K}[j] \subseteq \mathbb{R}^{n_{u}}$ - заданные выпуклые замкнутые конусы. Матрицы $A[j] \in \mathbb{R}^{n \times n}$ точно не заданы, но удовлетворяют ограничениям интервального типа

$$
A[j] \in \mathcal{A}[j]=\{A \mid \operatorname{Abs}(A-\tilde{A}[j]) \leq \hat{A}[j]\}, \quad j=1, \ldots, N .
$$

Матричные и векторные неравенства, а также операция тах ниже понимаются покомпонентно.

Множеством достижимости (МД) $\mathcal{X}[k]$ системы (1.1)-(1.4) в момент $k \in\{1, \ldots, N\}$ называем множество точек $x \in \mathbb{R}^{n}$, для каждой из которых существуют $x[0], u[\cdot]$ и $A[\cdot]$, удовлетворяющие (1.2)-(1.4) и порождающие решение $x[\cdot]$ системы (1.1) такое, что $x[k]=x$.

Полезно рассматривать также множества достижимости $\mathcal{Z}[k]$ системы (1.1), (1.3)-(1.7):

$$
\begin{gathered}
\mu[j]=\mu[j-1]-\|u[j]\|_{\infty}, \quad j=1, \ldots, N ; \\
\mu[j] \geq 0, \quad j=1, \ldots, N ; \\
z[0]=\{x[0], \mu[0]\} \in \mathcal{Z}_{0} \quad\left(\mathcal{Z}_{0} \subset \mathbb{R}^{n} \times\left[0, \mu_{0}\right]\right)
\end{gathered}
$$

в "расширенном" пространстве точек $z=\{x, \mu\}=\left(x^{\top}, \mu\right)^{\top} \in \mathbb{R}^{n+1}$, где $\mu$ соответствует текущему резерву $u$ и удовлетворяет фазовым ограничениям (1.6), которые накладываются вместо интегральных ограничений на $u$ из (1.2).

Множеством достижимости $\mathcal{Z}[k]=\mathcal{Z}\left(k, 0, \mathcal{Z}_{0}\right)$ системы $(1.1),(1.3)-(1.7)$ в момент $k \in$ $\{1, \ldots, N\}$ называем множество точек $z=\{x, \mu\} \in \mathbb{R}^{n+1}$, для каждой из которых существуют такие $z[0]=\{x[0], \mu[0]\}, u[\cdot]$ и $A[\cdot]$, удовлетворяющие $(1.3),(1.4),(1.7)$, что порождаемое ими в силу (1.1), (1.5) решение $z[\cdot]=\{x[\cdot], \mu[\cdot]\}$ будет удовлетворять условиям $z[k]=z$ и (1.6).

Для выявления связи между МД $\mathcal{Z}[k]$ и МД $\mathcal{X}[k]$ далее обычно будем полагать, что

$$
\mathcal{Z}_{0}=\mathcal{X}_{0} \times\left[0, \mu_{0}\right]=\left\{z=\{x, \mu\} \mid x \in \mathcal{X}_{0}, \mu \in\left[0, \mu_{0}\right]\right\} .
$$

Многозначные функции $\mathcal{X}[k], k=1, \ldots, N$, и $\mathcal{Z}[k], k=1, \ldots, N$, известны как трубки достижимости $\mathcal{X}[\cdot]$ и $\mathcal{Z}[\cdot]$.

Напомним определения объектов, которые будем использовать ниже.

Параллелепипедом $\mathcal{P}(p, P, \pi) \subset \mathbb{R}^{n}$ называем множество $\mathcal{P}=\mathcal{P}(p, P, \pi) \stackrel{\text { def }}{=}\{x \mid x=p+$ $\left.\sum_{i=1}^{n} p^{i} \pi_{i} \xi_{i},\|\xi\|_{\infty} \leq 1\right\}$, где $p \in \mathbb{R}^{n} ; P=\left\{p_{j}^{i}\right\}=\left\{p^{i}\right\} \in \mathbb{R}^{n \times n}$ - неособая матрица $(\operatorname{det} P \neq 0)$ со столбцами $p^{i}$ единичной длины (условие нормировки $\left\|p^{i}\right\|_{2}=1$ может быть опущено с целью упрощения формул); $\pi \in \mathbb{R}^{n}, \pi \geq 0$. Можно сказать, что $p$ - центр параллелепипеда, $P-$ матрица ориентации, $p^{i}$ - направления, $\pi_{i}-$ величины его "полуосей".

Параллелотопом $\mathcal{P}[p, \bar{P}] \subset \mathbb{R}^{n}$ называем множество $\mathcal{P}=\mathcal{P}[p, \bar{P}] \stackrel{\text { def }}{=}\{x \mid x=p+\bar{P} \zeta$, $\left.\|\zeta\|_{\infty} \leq 1\right\}$, где $p \in \mathbb{R}^{n}$, а матрица $\bar{P}=\left\{\bar{p}^{i}\right\} \in \mathbb{R}^{n \times m}, m \leq n$. Называем параллелотоп $\mathcal{P}$ невырожденным, если $m=n$ и $\operatorname{det} \bar{P} \neq 0$.

Каждый параллелепипед $\mathcal{P}(p, P, \pi)$ - это параллелотоп $\mathcal{P}[p, \bar{P}]$ с $\bar{P}=P \operatorname{diag} \pi ;$ каждый невырожденный параллелотоп - это параллелепипед с $P=\bar{P}, \pi=\mathrm{e}$.

Далее, как правило, считаем выполненным следующее предположение.

Предположение 1. Множество $\mathcal{X}_{0}$ - это параллелепипед: $\mathcal{X}_{0}=\mathcal{P}_{0}=\mathcal{P}\left(p_{0}, P_{0}, \pi_{0}\right)=$ $\mathcal{P}\left[p_{0}, \bar{P}_{0}\right] \subset \mathbb{R}^{n}$, а конусы $\mathcal{K}[j]$ таковы, что параллелепипедами являются множества

$$
\mathcal{R}[j] \stackrel{\text { def }}{=} \mathcal{C} \cap \mathcal{K}[j], \quad \mathcal{C}=\mathcal{P}(0, I, \mathrm{e}) \subset \mathbb{R}^{n_{u}} .
$$

Здесь $\mathcal{C}$ - единичный куб в $\mathbb{R}^{n_{u}}$ с центром в нуле. При этом МД $\mathcal{X}[k]$ и $\mathcal{Z}[k]$, вообще говоря, не являются параллелепипедами, а их точное построение оказывается непростой задачей.

Работа посвящена описанию МД $\mathcal{X}[k]$ и $\mathcal{Z}[k]$ и нахождению двусторонних полиэдралъных оценок $\mathcal{P}^{ \pm}[k]$ и $\Pi^{ \pm}[k]$ простой формы для $\mathcal{X}[k]$ и $\mathcal{Z}[k]$ и, следуя подходу из $[3 ; 7]$, описанию 
целых семейств таких оценок. Введение данных семейств позволяет более точно оценить МД в виде пересечения нескольких внешних оценок и объединения нескольких внутренних.

Для оценивания $\mathcal{Z}[k]$ используем класс политопов $\Pi=\Pi\left(\left\{\mathcal{P}^{\mathrm{b}}, 0\right\},\left\{\mathcal{P}^{\mathrm{t}}, \mu^{\mathrm{t}}\right\}\right) \subset \mathbb{R}^{n+1}$ специального типа [13], которые называем $\Pi$-политопами и которые определяются своими "нижним" и "верхним" $\mu$-сечениями $\mathcal{P}^{\mathrm{b}}$ и $\mathcal{P}^{\mathrm{t}}$ посредством операции выпуклой оболочки, где вышеупомянутые сечения - это либо параллелепипеды с одинаковыми матрицами ориентации, либо одинаковые параллелотопы (в последнем случае назовем такой $\Pi$-политоп $\Pi$-цилиндром):

$$
\begin{gathered}
\Pi=\Pi\left(\left\{\mathcal{P}^{\mathrm{b}}, 0\right\},\left\{\mathcal{P}^{\mathrm{t}}, \mu^{\mathrm{t}}\right\}\right) \stackrel{\text { def }}{=} \operatorname{co}\left(\left\{\mathcal{P}^{\mathrm{b}}, 0\right\} \cup\left\{\mathcal{P}^{\mathrm{t}}, \mu^{\mathrm{t}}\right\}\right), \quad \mu^{\mathrm{t}} \geq 0, \\
\mathcal{P}^{\mathrm{b}}=\mathcal{P}\left(p^{\mathrm{b}}, P^{\mathrm{b}}, \pi^{\mathrm{b}}\right), \quad \mathcal{P}^{\mathrm{t}}=\mathcal{P}\left(p^{\mathrm{t}}, P^{\mathrm{t}}, \pi^{\mathrm{t}}\right), \quad P^{\mathrm{b}}=P^{\mathrm{t}}=P \\
\text { или } \quad \mathcal{P}^{\mathrm{b}}=\mathcal{P}^{\mathrm{t}}=\mathcal{P}\left[p^{\mathrm{t}}, \bar{P}^{\mathrm{t}}\right] .
\end{gathered}
$$

Оценки $\Pi^{ \pm}[k]$ для $\mathcal{Z}[k]$ позволят найти оценки для $\mathcal{X}[k]$ в форме параллелотопов и параллелепипедов: $\mathcal{P}^{-}[k] \subseteq \mathcal{X}[k] \subseteq \mathcal{P}^{+}[k], \mathcal{P}^{-}[k]=\mathcal{P}\left[p^{-}[k], \bar{P}^{-}[k]\right], \mathcal{P}^{+}[k]=\mathcal{P}\left(p^{+}[k], P^{+}[k], \pi^{+}[k]\right)$.

Иногда будем считать выполненным следующее предположение.

Предположение 2. Все матрицы $\tilde{A}[j]$ из ограничения $(1.4)-$ неособые, m. е. $\operatorname{det} \tilde{A}[j] \neq$ $0, j=1, \ldots, N$.

\section{2. Точное описание множеств достижимости $\mathcal{X}[k]$ и $\mathcal{Z}[K]$}

Рассуждения, аналогичные [8, с.18-19], показывают, что МД $\mathcal{Z}[k]$ обладают полугрупповым свойством:

$$
\mathcal{Z}\left(k, 0, \mathcal{Z}_{0}\right)=\mathcal{Z}\left(k, i, \mathcal{Z}\left(i, 0, \mathcal{Z}_{0}\right)\right), \quad \forall k, i: 0 \leq i \leq k \leq N,
$$

где через $\mathcal{Z}\left(k, i, \mathcal{Z}_{i}\right)$ обозначено МД в момент $k \in\{i, \ldots, N\}$ системы (1.1), (1.3)-(1.7), рассматриваемой при $j=i, \ldots, N$ с начальными условиями $z[i]=\{x[i], \mu[i]\} \in \mathcal{Z}_{i}$.

Удобно искать МД $\mathcal{Z}[k]$ в виде объединения их $\mu$-сечений $\mathcal{X}(\mu, k)$ :

$$
\mathcal{Z}[k]=\bigcup_{0 \leq \mu \leq \mu^{\mathrm{t}}[k]}\{\mathcal{X}(\mu, k), \mu\} .
$$

Свойство (2.1) позволяет получить рекуррентные соотношения для $\mathcal{Z}[k]$, по форме напоминающие соотношения [13, (2.4)] для МД линейных систем, но пригодные для систем с неопределенностями в матрицах (1.4). В этих соотношениях будут фигурировать ограниченные множества типа

$$
\mathcal{Z}=\bigcup_{0 \leq \mu \leq \mu^{\mathrm{t}}}\{\mathcal{X}(\mu), \mu\} \subseteq \mathbb{R}^{n+1}
$$

и следующие операции с множествами $\mathcal{Z} \subseteq \mathbb{R}^{n+1}$, представленными в виде (2.3):

$$
\begin{gathered}
\mathcal{Z} \oplus v \stackrel{\text { def }}{=} \bigcup_{0 \leq \mu \leq \mu^{\mathrm{t}}}\{\mathcal{X}(\mu)+v, \mu\}, \quad \forall v \in \mathbb{R}^{n} ; \\
\mathcal{A} \otimes \mathcal{Z} \stackrel{\text { def }}{=} \bigcup_{0 \leq \mu \leq \mu^{\mathrm{t}}}\{\mathcal{A} \circ \mathcal{X}(\mu), \mu\} ; \\
\mathcal{Z} \uplus \mathcal{R} \stackrel{\text { def }}{=} \tilde{\mathcal{Z}}=\bigcup_{0 \leq \mu \leq \mu^{\mathrm{t}}}\{\tilde{\mathcal{X}}(\mu), \mu\}, \tilde{\mathcal{X}}(\mu)=\bigcup_{\mu \leq \zeta \leq \mu^{\mathrm{t}}}(\mathcal{X}(\zeta)+(\zeta-\mu) \mathcal{R}), \quad \forall \mathcal{R} \subset \mathbb{R}^{n} .
\end{gathered}
$$

Эти операции определяются с помощью операций с множествами в $\mathbb{R}^{n}$ : суммы Минковского $\mathcal{X}^{1}+\mathcal{X}^{2} \stackrel{\text { def }}{=}\left\{y \mid y=x^{1}+x^{2}, x^{k} \in \mathcal{X}^{k}\right\}$, оббединения множеств $\mathcal{X}^{1} \cup \mathcal{X}^{2}$ и умножения множества $\mathcal{X}$ на интервальную матричу $\mathcal{A}=\{A \mid \underline{A} \leq A \leq \bar{A}\}=\{A \mid \operatorname{Abs}(A-\tilde{A}) \leq \hat{A}\}$, где $\tilde{A}=(\underline{A}+\bar{A}) / 2$, $\hat{A}=(\bar{A}-\underline{A}) / 2: \mathcal{A} \circ \mathcal{X} \stackrel{\text { def }}{=}\left\{y \in \mathbb{R}^{n} \mid y=A x, A \in \mathcal{A}, x \in \mathcal{X}\right\}$. Таким образом, первые две операции 
в (2.4) действуют на каждое сечение независимо, а последняя комбинирует операции суммы Минковского и объединения по сечениям. Операции $\oplus$ и $\uplus$ были введены ранее в [13].

Будем говорить, что сечения множества $\mathcal{Z} \subseteq \mathbb{R}^{n+1}$ вида (2.2) не возрастают, если $\mathcal{X}\left(\mu^{1}\right) \supseteq \mathcal{X}\left(\mu^{2}\right)$ для любых $\mu^{1}, \mu^{2}$, таких что $0 \leq \mu^{1} \leq \mu^{2} \leq \mu^{\mathrm{t}}$.

Все операции из (2.4) обладают тем полезным свойством, что сохраняют невозрастание сечений - см. [13, лемма 2.1] и следующую лемму.

Лемма 1. Пусть множество $\mathcal{Z}$ имеет вид $\mathcal{Z}=\bigcup_{0 \leq \mu \leq \mu^{\mathrm{t}}}\{\mathcal{X}(\mu), \mu\} \subseteq \mathbb{R}^{n+1}$ и его сечения не возрастают. Тогда сечения $\mathcal{A} \otimes \mathcal{Z}$ также не возрастают.

Д о к а з а т е л ь с т в о очевидно: если $0 \leq \mu^{1} \leq \mu^{2} \leq \mu_{0}$, то по условию $\mathcal{X}\left(\mu^{1}\right) \supseteq \mathcal{X}\left(\mu^{2}\right)$, откуда $\mathcal{A} \circ \mathcal{X}\left(\mu^{1}\right)=\left\{A x \mid A \in \mathcal{A}, x \in \mathcal{X}\left(\mu^{1}\right)\right\} \supseteq\left\{A x \mid A \in \mathcal{A}, x \in \mathcal{X}\left(\mu^{2}\right)\right\}=\mathcal{A} \circ \mathcal{X}\left(\mu^{2}\right)$.

Теорема 1. Пусть $\mathcal{Z}[k]-$ - Д системы (1.1), (1.3)-(1.7) с начальным множеством $\mathcal{Z}_{0}$ типа $(2.3)$ с $\mu^{\mathrm{t}}=\mu_{0}$, причем $\mu$-сечения множества $\mathcal{Z}_{0}$ не возрастают. Тогда $\mathcal{Z}[k]$ определяются следуюшими рекуррентными соотношениями:

$$
\mathcal{Z}[k]=(\mathcal{A}[k] \otimes \mathcal{Z}[k-1] \oplus v[k]) \uplus B[k] \mathcal{R}[k], k=1, \ldots, N ; \quad \mathcal{Z}[0]=\mathcal{Z}_{0},
$$

и в (2.2) можно положить $\mu^{\mathrm{t}}[k]=\mu_{0}, k=1, \ldots, N$. При выполнении условия (1.8) оказывается, ито $\mathcal{X}[k]=\bigcup\left\{\mathcal{X}(\mu, k) \mid 0 \leq \mu \leq \mu_{0}\right\}=\mathcal{X}(0, k), k=1, \ldots, N$, т.е. МД $\mathcal{X}[k]$ системы (1.1)-(1.4) совпадают с "нижними" сечениями множеств $\mathcal{Z}[k]$, соответствующими $\mu=0$.

Д о к а з а т е л ь с т в о. Сначала докажем индукцией по $k$, что МД $\mathcal{Z}[k]$ системы (1.1), (1.3)-(1.7) с начальным множеством $\mathcal{Z}_{0}$ типа $(2.3)$ с $\mu^{\mathrm{t}}=\mu_{0}$ удовлетворяют соотношениям

$$
\mathcal{Z}[k]=(\mathcal{A}[k] \otimes \mathcal{Z}[k-1] \oplus v[k]) \uplus B[k](\partial \mathcal{C} \cap \mathcal{K}[k]), k=1, \ldots, N ; \quad \mathcal{Z}[0]=\mathcal{Z}_{0} .
$$

Пусть $Z[k]$ - МД системы (1.1), (1.3)-(1.7), представленные в виде (2.2). Обозначим $\mathcal{Z}^{*}[k]=$ $\bigcup_{0 \leq \mu \leq \mu^{*}[k]}\left\{\mathcal{X}^{*}(\mu, k), \mu\right\}=(\mathcal{A}[k] \otimes \mathcal{Z}[k-1] \oplus v[k]) \uplus B[k](\partial \mathcal{C} \cap \mathcal{K}[k]), \mu^{*}[k]=\mu_{0}$. Примем $\mathcal{Z}^{*}[0]=$ $\mathcal{Z}[0]=\mathcal{Z}_{0}$. Предположим, что доказали $\mu^{\mathrm{t}}[k-1]=\mu_{0}$ и $\mathcal{Z}^{*}[k-1]=\mathcal{Z}[k-1]$, и докажем такие же равенства для $k$, установив два противоположных включения.

Согласно определениям используемых операций и равенству $\mathcal{Z}^{*}[k-1]=\mathcal{Z}[k-1]$ сечение множества $\mathcal{Z}^{*}[k]$, соответствующее фиксированному $\mu$, имеет вид

$$
\mathcal{X}^{*}(\mu, k)=\bigcup_{\mu \leq \zeta \leq \mu^{\mathrm{t}}[k-1]\left(=\mu_{0}\right)}(\mathcal{A}[k] \circ \mathcal{X}(\zeta, k-1)+v[k]+(\zeta-\mu) B[k](\partial \mathcal{C} \cap \mathcal{K}[k])) .
$$

Пусть $z=\{x, \mu\} \in \mathcal{Z}[k]$. Ввиду полугруппового свойства (2.1) при $i=k-1$ эта точка получена из некоторой точки $z[k-1]=\{x[k-1], \mu[k-1]\} \in \mathcal{Z}[k-1]$ в силу системы (1.1), (1.3)-(1.6), т. е. найдутся такие $A[k] \in \mathcal{A}[k]$ и $u[k] \in \mathcal{K}[k]$, что $x=A[k] x[k-1]+B[k] u[k]+v[k], \mu=\mu[k-1]-$ $\|u[k]\|_{\infty}$, и, значит, $-\mu+\mu[k-1]=\|u[k]\|_{\infty} \geq 0$. При этом $u[k]$ удовлетворяет $u[k] \in(\mu[k-1]-$ $\mu) \partial \mathcal{C} \cap \mathcal{K}[k]=(\mu[k-1]-\mu)(\partial \mathcal{C} \cap \mathcal{K}[k])$, поскольку $\mathcal{K}[k]-$ конус. Итак, нашлись $A[k] \in \mathcal{A}[k]$ и $\zeta=\mu[k-1]$, где $\mu \leq \zeta=\mu[k-1] \leq \mu^{\mathrm{t}}[k-1]=\mu_{0}$, так что, действительно, вышеупомянутую точку $x$ можно представить в виде одной из точек множества $\mathcal{X}^{*}(\mu, k)$. Получили $\mathcal{Z}[k] \subseteq \mathcal{Z}^{*}[k]$.

Обратно, пусть $z=\{x, \mu\} \in \mathcal{Z}^{*}[k]$, т. е. в силу $(2.7)$ найдутся $A[k] \in \mathcal{A}[k], x[k-1] \in \mathcal{X}(\zeta, k-1)$ и $u^{*}[k]$, удовлетворяющее $u^{*}[k] \in(\zeta-\mu) \partial \mathcal{C}$ и $u^{*}[k] \in \mathcal{K}[k]$, такие что $x=A[k] x[k-1]+B[k] u^{*}[k]+$ $v[k], \mu \leq \zeta \leq \mu^{\mathrm{t}}[k-1]=\mu_{0}$. Включение $u^{*}[k] \in(\zeta-\mu) \partial \mathcal{C}$ означает, что $\left\|u^{*}[k]\right\|_{\infty}=\zeta-\mu$, т. е. $\mu=\zeta-\left\|u^{*}[k]\right\|_{\infty}$. Таким образом, можем считать, что точку $z=\{x, \mu\} \in \mathcal{Z}^{*}[k]$ получили из точки $\{x[k-1], \mu[k-1]\} \in \mathcal{Z}[k-1]$ с $\mu[k-1]=\zeta \in\left[\mu, \mu^{\mathrm{t}}[k-1]\right]$ с соблюдением всех условий $(1.1)$, (1.3)-(1.6), т. е. $\mathcal{Z}^{*}[k] \subseteq \mathcal{Z}[k]$. Желаемое равенство $\mathcal{Z}[k]=\mathcal{Z}^{*}[k]$ доказано.

Из (2.6) и определения операций (2.4) следует, что все $\mu^{\mathrm{t}}[k]=\mu^{\mathrm{t}}[0]=\mu_{0}$.

Невозрастание сечений $Z[k]$ выводим из свойств операций, участвующих в (2.6) (см. лемму 1 и [13, лемма 2.1]). Отсюда же, с учетом того что $\mathcal{K}[k]$ - конусы, ввиду [13, лемма 2.2] вытекает эквивалентность соотношений (2.5) и (2.6). 
Осталось проверить утверждения про множества $\mathcal{X}[k]$.

Пусть $x \in \mathcal{X}[k]$. Тогда существуют $x[0], A[\cdot]$ и $u[\cdot]$, удовлетворяющие (1.1)-(1.4) и порождающие траекторию $x[\cdot]$ с $x[k]=x$. Введем для нее соответствующую функцию $\mu[\cdot]$ по правилу (1.5) с $\mu[0]=\mu_{0}$. Тогда $\mu[k]=\mu_{0}-\sum_{j=1}^{k}\|u[j]\|_{\infty} \geq 0$, где выполнение условий $\mu[j] \geq 0$, $j=1, \ldots, k$ (т. е. фазовых ограничений (1.6)) обеспечивается интегральным ограничением из (1.2). Получаем $\{x[k], \mu[k]\} \in \mathcal{Z}[k]$ и, ввиду представлений $(2.2), x[k] \in \mathcal{X}(\mu[k], k)$. Отсюда $x[k] \in \bigcup_{0 \leq \mu \leq \mu_{0}} \mathcal{X}(\mu, k) \subseteq \mathcal{X}(0, k)$ (последнее - с учетом невозрастания сечений $\left.Z[k]\right)$.

Пусть, наоборот, $x \in \mathcal{X}(\mu, k)$ при некотором $\mu \in\left[0, \mu_{0}\right]$. Значит, имеется траектория $\{x[\cdot], \mu[\cdot]\}$ системы (1.1), (1.3)-(1.7) такая, что $\mu=\mu[k], x=x[k] \in \mathcal{X}(\mu[k], k)$, причем для соответствующего $u[\cdot]$ получаем в силу (1.5) и (1.6), что $\sum_{j=1}^{k}\|u[j]\|_{\infty}=\mu[0]-\mu[k] \leq \mu[0] \leq \mu_{0}$. Полагая $u[j]=0$ для $j>k$, условие на $u[\cdot]$ из (1.2) можно считать выполненным. Согласно определению множества $Z[k]$ и начальному условию (1.8) имеем $x[0] \in \mathcal{X}_{0}$. Поскольку выполнены все условия (1.1)-(1.4), получаем $x=x[k] \in \mathcal{X}[k]$. Следовательно, доказали, что $\mathcal{X}(\mu, k) \subseteq \mathcal{X}[k]$, и, в связи с произвольностью $\mu \in\left[0, \mu_{0}\right]$, что $\mathcal{X}(0, k) \subseteq \mathcal{X}[k]$ и $\bigcup_{0 \leq \mu \leq \mu_{0}} \mathcal{X}(\mu, k) \subseteq \mathcal{X}[k]$.

Заметим, что множества $\mathcal{Z}[k]$ и $\mathcal{X}[k]$ могут быть невыпуклы ввиду присутствия операций $\otimes$ и о преобразования множеств с помощью интервальных матриц (см. [18]).

\section{3. Элементарные оценки}

При построении оценок $\Pi^{ \pm}[k]$ для $\mathcal{Z}[k]$ и $\mathcal{P}^{ \pm}[k]$ для $\mathcal{X}[k]$ мы используем свойства операций с множествами, упомянутых в разд. 2, и элементарные полиэдральные оценки для результатов таких операций. В $[13 ; 18]$ указаны способы построения ряда элементарных оценок. Кратко напомним их.

Опорные функции параллелепипеда и параллелотопа вычисляются по следующим формулам: $\rho(l \mid \mathcal{P}(p, P, \pi))=l^{\top} p+\left(\operatorname{Abs}\left(l^{\top} P\right)\right) \pi, \rho(l \mid \mathcal{P}[p, \bar{P}])=l^{\top} p+\left(\operatorname{Abs}\left(l^{\top} \bar{P}\right)\right)$ e.

В ряде случаев полезно использовать эквивалентное представление параллелепипеда $\mathcal{P}=$ $\mathcal{P}(p, P, \pi): \mathcal{P}=\mathcal{P}\left(P, \gamma^{(-)}, \gamma^{(+)}\right)=\left\{x \mid \gamma^{(-)} \leq P^{-1} x \leq \gamma^{(+)}\right\}$, где $\gamma_{i}^{( \pm)}= \pm \rho\left( \pm\left(P^{-1}\right)^{\top} \mathrm{e}^{i} \mid \mathcal{P}\right), i=$ $1, \ldots, n$, и имеются следующие связи между параметрами, определяющими параллелепипед: $\gamma^{( \pm)}=P^{-1} p \pm \pi ; p=P\left(\gamma^{(-)}+\gamma^{(+)}\right) / 2, \pi=\left(\gamma^{(+)}-\gamma^{(-)}\right) / 2$.

Афинное преобразование параллелотопа - это параллелотоп: $A \mathcal{P}[p, \bar{P}]+a=\mathcal{P}[A p+a, A \bar{P}]$ для $A \in \mathbb{R}^{n \times r}, p \in \mathbb{R}^{r}, \bar{P} \in \mathbb{R}^{r \times r}, a \in \mathbb{R}^{n} ; A \mathcal{P}(p, P, \pi)=\mathcal{P}(A p, A P, \pi)$, если $\operatorname{det} A \neq 0$.

Рассмотрим способы построения элементарных внешних оценок и начнем с оценок в $\mathbb{R}^{n}$.

Определяем $\mathcal{P}$ как внешнюю (внутреннюю) оценку множества $\mathcal{Q} \subset \mathbb{R}^{n}$, если $\mathcal{Q} \subseteq \mathcal{P}$ $(\mathcal{P} \subseteq \mathcal{Q})$. Оценку называют тугой (в направлении $l$ ) [3, с. 91], если $\rho( \pm l \mid \mathcal{P})=\rho( \pm l \mid \mathcal{Q})$. Называем параллелепипед $\mathcal{P}\left(p^{+}, P^{+}, \pi^{+}\right)$внешней касающейся оченкой для $\mathcal{Q}$ и обозначаем ее как $\boldsymbol{P}_{P^{+}}^{+}(\mathcal{Q})$, если она является тугой в направлении $n$ следующих векторов (определяющих нормали к граням параллелепипеда): $l^{i}=\left(P^{+}\right)^{-1^{\top}} \mathrm{e}^{i}, i=1, \ldots, n$.

Касающаяся оценка $\boldsymbol{P}_{P^{+}}^{+}(\mathcal{Q})$ для $\mathcal{Q}$ с заданной матрицей ориентации $P^{+}$строится на основе значений опорной функции для $\mathcal{Q}: \boldsymbol{P}_{P^{+}}^{+}(\mathcal{Q})=\mathcal{P}\left(P^{+}, \gamma^{+(-)}, \gamma^{+(+)}\right), \gamma_{i}^{+( \pm)}= \pm \rho\left( \pm\left(P^{+}\right)^{-1^{\top}} \mathrm{e}^{i} \mid \mathcal{Q}\right)$. Матрицы $P^{+}$выступают как параметр, определяющий семейство оценок.

Касающиеся оценки для суммы двух параллелепипедов находятся по явной формуле: $\boldsymbol{P}_{P^{+}}^{+}\left(\sum_{k=1}^{2} \mathcal{P}\left(p^{k}, P^{k}, \pi^{k}\right)\right)=\mathcal{P}\left(\sum_{k=1}^{2} p^{k}, P^{+}, \sum_{k=1}^{2}\left(\operatorname{Abs}\left(\left(P^{+}\right)^{-1} P^{k}\right)\right) \pi^{k}\right)$, а для параллелотопов $\boldsymbol{P}_{P^{+}}^{+}\left(\sum_{k=1}^{2} \mathcal{P}\left[p^{k}, \bar{P}^{k}\right]\right)=\mathcal{P}\left[\sum_{k=1}^{2} p^{k}, P^{+} \operatorname{diag}\left(\sum_{k=1}^{2}\left(\operatorname{Abs}\left(\left(P^{+}\right)^{-1} \bar{P}^{k}\right)\right) \mathrm{e}\right)\right]$.

Оценки $\boldsymbol{P}_{P^{+}}^{+}\left(\mathcal{P}^{1} \cup \mathcal{P}^{2}\right)$ для объединения параллелотопов $\mathcal{P}^{k}=\mathcal{P}\left[p^{k}, \bar{P}^{k}\right]$ определяются формулой, вытекающей из соотношения $\rho\left(l \mid \mathcal{P}^{1} \cup \mathcal{P}^{2}\right)=\max _{1 \leq k \leq 2} \rho\left(l \mid \mathcal{P}^{k}\right)$ :

$$
\boldsymbol{P}_{P^{+}}^{+}\left(\mathcal{P}^{1} \cup \mathcal{P}^{2}\right)=\mathcal{P}\left(P^{+}, \gamma^{+(-)}, \gamma^{+(+)}\right), \gamma^{+( \pm)}= \pm \max _{1 \leq k \leq 2}\left\{ \pm\left(P^{+}\right)^{-1} p^{k}+\left(\operatorname{Abs}\left(\left(P^{+}\right)^{-1} \bar{P}^{k}\right)\right) \mathrm{e}\right\}
$$

Для нахождения оценки $\boldsymbol{P}_{P^{+}}^{+}(\mathcal{Q})$ для $\mathcal{Q}=\mathcal{A} \circ \mathcal{P}$, где $\mathcal{A}$ - интервальная матрица, $\mathcal{P}-$ 
параллелепипед, применимо любое из двух следующих выражений для опорной функции [18]:

$$
\rho(l \mid \mathcal{Q})=\max _{x \in \mathbb{E}(\mathcal{P})}\left\{l^{\top} \tilde{A} x+(\operatorname{Abs} l)^{\top} \hat{A}(\operatorname{Abs} x)\right\}, \quad \rho(l \mid \mathcal{Q})=\max _{A \in \mathbb{E}(\mathcal{A})}\left\{l^{\top} A p+\left(\operatorname{Abs}\left(l^{\top} A P\right)\right) \pi\right\},
$$

где $\mathbb{E}(\mathcal{P})$ и $\mathbb{E}(\mathcal{A})$ обозначают множества всех вершин $\mathcal{P}$ и $\mathcal{A}$, (т. е. множество точек $p+$ $\sum_{i=1}^{m} p^{i} \pi_{i} \xi_{i}$ с $\xi_{i} \in\{-1,1\}$ и множество матриц с элементами $\left.a_{i}^{j} \in\left\{\underline{a}_{i}^{j}, \bar{a}_{i}^{j}\right\}\right)$.

Перейдем к построению элементарных внешних оценок в $\mathbb{R}^{n+1}$ в виде $\Pi$-политопов.

Лемма 2. Пусть $\mathcal{Z}=\operatorname{co}\left(\mathcal{Z}^{\mathrm{b}} \cup \mathcal{Z}^{\mathrm{t}}\right)$, әде $\mathcal{Z}^{\mathrm{b}}=\left\{\mathcal{X}^{\mathrm{b}}, 0\right\}, \mathcal{Z}^{\mathrm{t}}=\left\{\mathcal{X}^{\mathrm{t}}, \mu^{\mathrm{t}}\right\}$, a $\mathcal{X}^{\mathrm{b}}$ и $\mathcal{X}^{\mathrm{t}}$ выпукльь. Тогда $\mathcal{Z}=\bigcup_{0 \leq \alpha \leq 1}\left(\alpha \mathcal{Z}^{\mathrm{t}}+(1-\alpha) \mathcal{Z}^{\mathrm{b}}\right)=\bigcup_{0 \leq \mu \leq \mu^{\mathrm{t}}}\{\mathcal{X}(\mu), \mu\}$, где $\mathcal{X}(\mu)=\alpha \mathcal{X}^{\mathrm{t}}+(1-\alpha) \mathcal{X}^{\mathrm{b}}, \alpha=\mu / \mu^{\mathrm{t}}$.

Д ок а з а т е ль с т в о. Если $z \in \alpha \mathcal{Z}^{\mathrm{t}}+(1-\alpha) \mathcal{Z}^{\mathrm{b}}$ при $\alpha \in[0,1]$, то, очевидно, $z \in \mathcal{Z}$. Пусть, наоборот, $z \in \mathcal{Z}$, т.е. $z=\sum_{i=1}^{m} \lambda_{i} z^{i}+\sum_{i=m+1}^{l} \lambda_{i} z^{i}$, где все $\lambda_{i} \geq 0 ; \sum_{i=1}^{l} \lambda_{i}=1 ; z^{i} \in \mathcal{Z}^{\mathrm{t}}$, $i=1, \ldots, m ; z^{i} \in \mathcal{Z}^{\mathrm{b}}, i=m+1, \ldots, l$. Положим $\alpha=\sum_{i=1}^{m} \lambda_{i}$. Если $\alpha \neq 0$ и $\alpha \neq 1$, то получим $z=\alpha z^{\mathrm{t}}+(1-\alpha) z^{\mathrm{b}}$, где $z^{\mathrm{t}}=\sum_{i=1}^{m} \lambda_{i} z^{i} /\left(\sum_{i=1}^{m} \lambda_{i}\right) \in \mathcal{Z}^{\mathrm{t}}, z^{\mathrm{b}}=\sum_{i=m+1}^{l} \lambda_{i} z^{i} /\left(\sum_{i=m+1}^{l} \lambda_{i}\right) \in \mathcal{Z}^{\mathrm{b}}$. Если $\alpha=1$, то $\sum_{i=m+1}^{l} \lambda_{i}=0$ и $z=\sum_{i=1}^{m} \lambda_{i} z^{i} \in \mathcal{Z}^{\mathrm{t}}$, Аналогично $z \in \mathcal{Z}^{\mathrm{b}}$ при $\alpha=0$.

Из [13, следствие 3.1] вытекают следующие, более детальные, представления П -политопа.

Следствие 1. Для П-политопа (1.10) с параллелепипедозначными сечениями имеем

$$
\begin{gathered}
\Pi=\bigcup_{0 \leq \mu \leq \mu^{\mathrm{t}}}\{\mathcal{P}(\mu), \mu\}, \quad \mathcal{P}(\mu)=\mathcal{P}(p(\mu), P, \pi(\mu))=\mathcal{P}\left(P, \gamma^{(-)}(\mu), \gamma^{(+)}(\mu)\right), \\
p(\mu)=\alpha p^{\mathrm{t}}+(1-\alpha) p^{\mathrm{b}}, \pi(\mu)=\alpha \pi^{\mathrm{t}}+(1-\alpha) \pi^{\mathrm{b}}, \gamma^{( \pm)}(\mu)=\alpha \gamma^{(+) \mathrm{t}}+(1-\alpha) \gamma^{(+) \mathrm{b}}, \alpha=\mu / \mu^{\mathrm{t}} ; \\
\rho(l \mid \mathcal{P}(\mu))=\alpha \rho\left(l \mid \mathcal{P}^{\mathrm{t}}\right)+(1-\alpha) \rho\left(l \mid \mathcal{P}^{\mathrm{b}}\right), \alpha=\mu / \mu^{\mathrm{t}}, \quad \forall l \in \mathbb{R}^{n},
\end{gathered}
$$

где использованы обозначения для представлений $\mathcal{P}^{\mathrm{i}}=\mathcal{P}\left(P, \gamma^{(-) \mathrm{i}}, \gamma^{(+) \mathrm{i}}\right), \mathrm{i}=\mathrm{b}, \mathrm{t}$.

Для ограниченного множества $\mathcal{Z} \subset \mathbb{R}^{n}$ вида (2.3) и фиксированной неособой матрицы $P^{+} \in \mathbb{R}^{n \times n}$ можно построить $\Pi$-политоп $\Pi^{+}$вида

$$
\Pi^{+}=\Pi_{P^{+}}^{+}(\mathcal{Z}) \stackrel{\text { def }}{=} \Pi\left(\left\{\mathcal{P}^{+\mathrm{b}}, 0\right\},\left\{\mathcal{P}^{+\mathrm{t}}, \mu^{+\mathrm{t}}\right\}\right), \quad \mathcal{P}^{+\mathrm{b}}=\boldsymbol{P}_{P^{+}}^{+}\left(\mathcal{X}^{\mathrm{b}}\right), \mathcal{P}^{+\mathrm{t}}=\boldsymbol{P}_{P^{+}}^{+}\left(\mathcal{X}^{\mathrm{t}}\right), \mu^{+\mathrm{t}}=\mu^{\mathrm{t}},
$$

где $\mathcal{X}^{\mathrm{i}}=\mathcal{X}\left(\mu^{\mathrm{i}}\right), \mathrm{i}=\mathrm{b}, \mathrm{t},-$ соответствующие $\mu$-сечения $\mathcal{Z}$, а $\boldsymbol{P}_{P^{+}}^{+}\left(\mathcal{X}^{\mathrm{i}}\right)-$ внешние касающиеся оценки для них. При этом $\Pi^{+}$не обязательно оказывается внешней оценкой для $\mathcal{Z}$.

Называем $\Pi$-политоп внешней касающейся оченкой для $\mathcal{Z} \subset \mathbb{R}^{n+1}$, если $\mathcal{Z} \subseteq \Pi$ и все $\mu$-сечения политопа $П$ являются касающимися оценками для $\mu$-сечений множества $\mathcal{Z}$.

Лемма 3. Пусть $\mathcal{Z}=\operatorname{co}\left(\mathcal{Z}^{\mathrm{b}} \cup \mathcal{Z}^{\mathrm{t}}\right)$ ограничено и выполнены условия леммы 2. Тогда $\Pi$ политоп вида (3.3) является внешней касающейся оченкой для $\mathcal{Z}$ при любой матрице ориентации $P^{+}$. При этом если $\mathcal{X}^{\mathrm{b}} \supseteq \mathcal{X}^{\mathrm{t}}$, то н-сечения $\mathcal{Z}$ и $\Pi^{+}$не возрастают.

Д о к а з а т е л ь с т в о. В силу леммы 2 , свойств опорных функций и следствия 1 имеем для $\mu$-сечений $\mathcal{X}(\mu)$ и $\mathcal{P}^{+}(\mu)$ множеств $\mathcal{Z}$ и $\Pi^{+}$для любого $l \in \mathbb{R}^{n}: \rho(l \mid \mathcal{X}(\mu))=\alpha \rho\left(l \mid \mathcal{X}^{\mathrm{t}}\right)+$ $(1-\alpha) \rho\left(l \mid \mathcal{X}^{\mathrm{b}}\right) \leq \alpha \rho\left(l \mid \mathcal{P}^{+\mathrm{t}}\right)+(1-\alpha) \rho\left(l \mid \mathcal{P}^{+\mathrm{b}}\right)=\rho\left(l \mid \mathcal{P}^{+}(\mu)\right)$, т. е. $\mathcal{Z} \subseteq \Pi^{+}$. Обозначим значения опорной функции произвольного множества $\mathcal{X} \subset \mathbb{R}^{n}$ на векторах $\pm\left(P^{+}\right)^{-1^{\top}} \mathrm{e}^{i}$ через $\rho_{i}^{ \pm}(\mathcal{X})$. Тогда в силу того что $\mathcal{P}^{+\mathrm{b}}$ и $\mathcal{P}^{+\mathrm{t}}-$ касающиеся оценки для $\mathcal{X}^{\mathrm{b}}$ и $\mathcal{X}^{\mathrm{t}}$, получаем $\rho_{i}^{ \pm}(\mathcal{X}(\mu))=$ $\alpha \rho_{i}^{ \pm}\left(\mathcal{X}^{\mathrm{t}}\right)+(1-\alpha) \rho_{i}^{ \pm}\left(\mathcal{X}^{\mathrm{b}}\right)=\alpha \rho_{i}^{ \pm}\left(\mathcal{P}^{+\mathrm{t}}\right)+(1-\alpha) \rho_{i}^{ \pm}\left(\mathcal{P}^{+\mathrm{b}}\right)=\rho_{i}^{ \pm}\left(\mathcal{P}^{+}(\mu)\right)$, т. е. $\Pi^{+}-$касающаяся оценка для $\mathcal{Z}$. Из вышеприведенных выражений видно, что $\rho(l \mid \mathcal{X}(\mu))$ и $\rho\left(l \mid \mathcal{P}^{+}(\mu)\right)$ являются линейными функциями по $\mu$ (т.к. $\left.\alpha=\mu / \mu^{\mathrm{t}}\right)$. При этом если $\mathcal{X}^{\mathrm{b}} \supseteq \mathcal{X}^{\mathrm{t}}$, то производная по $\mu$ неположительна: $\frac{d}{d \mu} \rho(l \mid \mathcal{X}(\mu))=\left(1 / \mu^{\mathrm{t}}\right)\left(\rho\left(l \mid \mathcal{X}^{\mathrm{t}}\right)-\rho\left(l \mid \mathcal{X}^{\mathrm{b}}\right)\right) \leq 0$, т. е. функция не возрастает при любом $l$ и выпуклые $\mu$-сечения $\mathcal{Z}$ не возрастают. Аналогичное заключение делаем относительно невозрастания сечений $\Pi^{+}$, поскольку здесь все определяется только значениями $\rho_{i}^{ \pm}\left(\mathcal{P}^{+}(\mu)\right)$, $i=1, \ldots, n, \mathrm{a} \frac{d}{d \mu} \rho_{i}^{ \pm}\left(\mathcal{P}^{+}(\mu)\right)=\left(1 / \mu^{\mathrm{t}}\right)\left(\rho_{i}^{ \pm}\left(\mathcal{X}^{\mathrm{t}}\right)-\rho_{i}^{ \pm}\left(\mathcal{X}^{\mathrm{b}}\right)\right) \leq 0$. 
Лемма 4. Пусть $\mathcal{Z}=\operatorname{co}\left(\mathcal{Z}^{\mathrm{b}} \cup \mathcal{Z}^{\mathrm{t}}\right)=\bigcup_{0 \leq \mu \leq \mu^{\mathrm{t}}}\{\mathcal{X}(\mu), \mu\}$, где $\mathcal{Z}^{\mathrm{b}}=\left\{\mathcal{X}^{\mathrm{b}}, 0\right\}, \mathcal{Z}^{\mathrm{t}}=\left\{\mathcal{X}^{\mathrm{t}}, \mu^{\mathrm{t}}\right\}$, множества $\mathcal{X}^{\mathrm{b}}$ и $\mathcal{X}^{\mathrm{t}}$ выпуклы, и пусть $\mathcal{R} \subset \mathbb{R}^{n}-$ произвольное выпуклое множество. Тогда множество $\tilde{\mathcal{Z}}=\mathcal{Z} \uplus \mathcal{R}$ из (2.4), множество $\hat{\mathcal{Z}} \stackrel{\text { def }}{=} \bigcup_{0 \leq \mu \leq \mu^{t}}\{\hat{\mathcal{X}}(\mu), \mu\}, \hat{\mathcal{X}}(\mu)=\operatorname{co}\left(\mathcal{X}(\mu) \cup\left(\mathcal{X}^{\mathrm{t}}+\right.\right.$ $\left.\left.\left(\mu^{\mathrm{t}}-\mu\right) \mathcal{R}\right)\right)$ имножество $\check{\mathcal{Z}} \stackrel{\text { def }}{=} \operatorname{co}\left(\check{\mathcal{Z}}^{\mathrm{b}} \cup \check{\mathcal{Z}}^{\mathrm{t}}\right)=\operatorname{co}\left(\left\{\operatorname{co}\left(\mathcal{X}^{\mathrm{b}} \cup\left(\mathcal{X}^{\mathrm{t}}+\mu^{\mathrm{t}} \mathcal{R}\right)\right), 0\right\} \cup\left\{\mathcal{X}^{\mathrm{t}}, \mu^{\mathrm{t}}\right\}\right)$ cовпадают.

Д о к а з а т е л ь с т в о. Проверим, что $\hat{\mathcal{Z}} \subseteq \tilde{\mathcal{Z}}, \check{\mathcal{Z}} \subseteq \tilde{\mathcal{Z}}$. Очевидно, $\mathcal{X}(\mu) \cup\left(\mathcal{X}^{\mathrm{t}}+\left(\mu^{\mathrm{t}}-\right.\right.$ $\mu) \mathcal{R}) \subseteq \tilde{\mathcal{X}}(\mu)$. Поскольку $\tilde{\mathcal{Z}}$ при наших предположениях выпукло (см. [13, лемма 2.3]), имеем $\hat{\mathcal{X}}(\mu)=\operatorname{co}\left(\mathcal{X}(\mu) \cup\left(\mathcal{X}^{\mathrm{t}}+\left(\mu^{\mathrm{t}}-\mu\right) \mathcal{R}\right)\right) \subseteq \operatorname{co~} \tilde{\mathcal{X}}(\mu)=\tilde{\mathcal{X}}(\mu)$, откуда $\hat{\mathcal{Z}} \subseteq \tilde{\mathcal{Z}}$. С учетом выпуклости $\check{\mathcal{Z}}$ и совпадения верхних и нижних $\mu$-сечений $\breve{\mathcal{Z}}$ и $\hat{\mathcal{Z}}$ получаем $\check{\mathcal{Z}}=\operatorname{co~} \check{\mathcal{Z}} \subseteq \operatorname{co} \hat{\mathcal{Z}} \subseteq \operatorname{co~} \tilde{\mathcal{Z}}=\tilde{\mathcal{Z}}$.

Проверим, что $\tilde{\mathcal{Z}} \subseteq \hat{\mathcal{Z}}$. Пусть $x \in \tilde{\mathcal{X}}(\mu)$, т. е. найдутся такие $\zeta \in\left[\mu, \mu^{\mathrm{t}}\right], x^{0} \in \mathcal{X}(\zeta), x^{1} \in \mathcal{R}$, что $x=x^{0}+(\zeta-\mu) x^{1}$. В силу леммы 2 найдутся $x^{2} \in \mathcal{X}^{\mathrm{t}}$ и $x^{3} \in \mathcal{X}^{\mathrm{b}}$ такие, что можем записать $x=\left(\zeta / \mu^{\mathrm{t}}\right) x^{2}+\left(\left(\mu^{\mathrm{t}}-\zeta\right) / \mu^{\mathrm{t}}\right) x^{3}+(\zeta-\mu) x^{1}$. Обозначим $\alpha=(\zeta-\mu) /\left(\mu^{\mathrm{t}}-\mu\right)$. Очевидно, $\alpha \in[0,1]$. Элементарными выкладками проверяется, что можно представить $x$ в виде $x=\tilde{x}$, где $\tilde{x}=(1-\alpha) \tilde{x}^{3}+\alpha \tilde{x}^{2}, \tilde{x}^{3}=\left(\mu / \mu^{\mathrm{t}}\right) x^{2}+\left(\left(\mu^{\mathrm{t}}-\mu\right) / \mu^{\mathrm{t}}\right) x^{3}, \tilde{x}^{2}=x^{2}+\left(\mu^{\mathrm{t}}-\mu\right) x^{1}$. Но, очевидно, $\tilde{x}^{2} \in \mathcal{X}^{\mathrm{t}}+\left(\mu^{\mathrm{t}}-\mu\right) \mathcal{R}$, a $\tilde{x}^{3} \in \mathcal{X}(\mu)$ в силу леммы 2. Поэтому $x \in \operatorname{co}\left(\mathcal{X}(\mu) \cup\left(\mathcal{X}^{\mathrm{t}}+\left(\mu^{\mathrm{t}}-\mu\right) \mathcal{R}\right)\right)=\hat{\mathcal{X}}(\mu)$.

Убедимся, что $\tilde{\mathcal{Z}} \subseteq \check{\mathcal{Z}}$. Пусть $z=\{x, \mu\} \in \tilde{\mathcal{Z}}$, т. е. $\mu \in\left[0, \mu^{\mathrm{t}}\right]$, а $x \in \tilde{\mathcal{X}}(\mu)=\hat{\mathcal{X}}(\mu)$ (использовали уже доказанное равенство $\tilde{\mathcal{Z}}=\hat{\mathcal{Z}})$. Значит, найдутся такие $x^{\mu} \in \mathcal{X}(\mu), x^{1, \mathrm{t}} \in \mathcal{X}^{\mathrm{t}}, x^{\mathcal{R}} \in \mathcal{R}$ и $\beta \in[0,1]$, что $x=\beta x^{\mu}+(1-\beta)\left(x^{1, \mathrm{t}}+\left(\mu^{\mathrm{t}}-\mu\right) x^{\mathcal{R}}\right)$. В силу леммы 2 точка $z^{\mu}=\left\{x^{\mu}, \mu\right\}$ может быть представлена в таком виде, что $x^{\mu}=\alpha x^{2, \mathrm{t}}+(1-\alpha) x^{2, \mathrm{~b}}, \mu=\alpha \mu^{\mathrm{t}}$, где $x^{2, \mathrm{t}} \in \mathcal{X}^{\mathrm{t}}, x^{2, \mathrm{~b}} \in \mathcal{X}^{\mathrm{b}}$, $\alpha \in[0,1]$. Тогда получается, что $x=\beta\left(\alpha x^{2, \mathrm{t}}+(1-\alpha) x^{2, \mathrm{~b}}\right)+(1-\beta)\left(x^{1, \mathrm{t}}+\mu^{\mathrm{t}}(1-\alpha) x^{\mathcal{R}}\right)=$ $(1-\beta)(1-\alpha)\left(x^{1, \mathrm{t}}+\mu^{\mathrm{t}} x^{\mathcal{R}}\right)+\beta(1-\alpha) x^{2, \mathrm{~b}}+\alpha\left(\beta x^{2, \mathrm{t}}+(1-\beta) x^{1, \mathrm{t}}\right)$ (добавили и вычли $(1-\beta) \alpha x^{1, \mathrm{t}}$ и перегруппировали члены). Вводя новые обозначения, имеем $x=(1-\alpha) \check{x}^{\mathrm{b}}+\alpha x^{3, \mathrm{t}}$, где $\check{x}^{\mathrm{b}}=\beta x^{2, \mathrm{~b}}+(1-\beta)\left(x^{1, \mathrm{t}}+\mu^{\mathrm{t}} x^{\mathcal{R}}\right) \in \check{\mathcal{X}}^{\mathrm{b}}=\operatorname{co}\left(\mathcal{X}^{\mathrm{b}} \cup\left(\mathcal{X}^{\mathrm{t}}+\mu^{\mathrm{t}} \mathcal{R}\right)\right), x^{3, \mathrm{t}}=\beta x^{2, \mathrm{t}}+(1-\beta) x^{1, \mathrm{t}} \in \mathcal{X}^{\mathrm{t}}$. Учитывая, что $\alpha=\mu / \mu^{\mathrm{t}}$, получаем $z=\{x, \mu\} \in \operatorname{co}\left(\left\{\operatorname{co}\left(\mathcal{X}^{\mathrm{b}} \cup\left(\mathcal{X}^{\mathrm{t}}+\mu^{\mathrm{t}} \mathcal{R}\right)\right), 0\right\} \cup\left\{\mathcal{X}^{\mathrm{t}}, \mu^{\mathrm{t}}\right\}\right)=\check{\mathcal{Z}}$.

Утверждение 1. Пусть $\mathcal{Z}=\operatorname{co}\left(\left\{\mathcal{X}^{\mathrm{b}}, 0\right\} \cup\left\{\mathcal{X}^{\mathrm{t}}, \mu^{\mathrm{t}}\right\}\right), \mu^{\mathrm{t}} \geq 0, \tilde{\mathcal{Z}}=\mathcal{Z} \uplus \mathcal{R}$, и множества $\mathcal{X}^{\mathrm{b}}$, $\mathcal{X}^{\mathrm{t}}, \mathcal{R} \subset \mathbb{R}^{n}$ ограничены и выпуклы. Тогда $\Pi$-политоп $\Pi^{+}=\Pi_{P^{+}}^{+}(\tilde{\mathcal{Z}})$, построенный для $\tilde{\mathcal{Z}}$ по формулам (3.3), является внешней касающейся оченкой для $\mathcal{Z} \uplus \mathcal{R}$ при любой неособой матрице ориентации $P^{+}$и имеем $\mathcal{P}^{+\mathrm{t}}=\boldsymbol{P}_{P^{+}}^{+}\left(\mathcal{X}^{\mathrm{t}}\right), \mu^{+\mathrm{t}}=\mu^{\mathrm{t}}, \mathcal{P}^{+\mathrm{b}}=\boldsymbol{P}_{P^{+}}^{+}\left(\mathcal{X}^{\mathrm{b}} \cup\left(\mathcal{X}^{\mathrm{t}}+\mu^{\mathrm{t}} \mathcal{R}\right)\right)$. Если вдобавок $\mathcal{X}^{\mathrm{b}} \supseteq \mathcal{X}^{\mathrm{t}}$ либо $0 \in \mathcal{R}$, то сечения множеств $\tilde{\mathcal{Z}} u \boldsymbol{\Pi}_{P^{+}}^{+}(\tilde{\mathcal{Z}})$ будут невозрастаюоиим.

Д о к а з а т е л ь с т в о. Доказанное в лемме 4 равенство $\tilde{\mathcal{Z}}=\check{\mathcal{Z}}$ дает указанную выше формулу для $\mathcal{P}^{+\mathrm{b}}$ и с учетом леммы 3 обеспечивает, что $\Pi^{+}$является внешней касающейся оценкой для $\tilde{\mathcal{Z}}$. Невозрастание сечений следует из второй части леммы 3 ввиду включений $\tilde{\mathcal{X}}^{\mathrm{t}} \subseteq \tilde{\mathcal{X}}^{\mathrm{b}}$, вытекающих из наложенных условий, поскольку при $\mathcal{X}^{\mathrm{b}} \supseteq \mathcal{X}^{\mathrm{t}}$ имеем $\tilde{\mathcal{X}}^{\mathrm{t}}=\mathcal{X}^{\mathrm{t}} \subseteq$ $\mathcal{X}^{\mathrm{b}} \subseteq \operatorname{co}\left(\mathcal{X}^{\mathrm{b}} \cup\left(\mathcal{X}^{\mathrm{t}}+\mu^{\mathrm{t}} \mathcal{R}\right)\right)=\tilde{\mathcal{X}}^{\mathrm{b}}$, а при $0 \in \mathcal{R}$, очевидно, $\tilde{\mathcal{X}}^{\mathrm{t}}=\mathcal{X}^{\mathrm{t}} \subseteq \mathcal{X}^{\mathrm{t}}+\mu^{\mathrm{t}} \mathcal{R} \subseteq \tilde{\mathcal{X}}^{\mathrm{b}}$.

Применяя следствие 1 , формулы для опорной функции параллелепипеда и (3.1), получаем

Следствие 2. Пусть П - это П-политоп (1.10) с параллелепипедозначными сечениями, $B \in \mathbb{R}^{n \times m}$ u $\mathcal{R}=\mathcal{P}(r, R, \rho)$ - параллелепипед в $\mathbb{R}^{m}$. Тогда внешней касаюшейся оценкой для $\Pi \uplus B \mathcal{R}$ с прочзвольной матричей ориентачии $P^{+}$является политоп $\Pi^{+}=\Pi_{P^{+}}^{+}(\Pi \uplus B \mathcal{R})$, построенный по правилу (3.3), причем его сечения определяются формулами

$$
\begin{gathered}
\mathcal{P}^{+}(\mu)=\mathcal{P}\left(P^{+}, \gamma^{+(-)}(\mu), \gamma^{+(+)}(\mu)\right), \quad 0 \leq \mu \leq \mu^{\mathrm{t}}, \\
\gamma_{i}^{+( \pm)}(\mu)=\left\{\begin{array}{l}
\alpha\left(D p^{\mathrm{t}} \pm C \pi^{\mathrm{t}}\right)_{i}+(1-\alpha)\left(D p^{\mathrm{b}} \pm C \pi^{\mathrm{b}}\right)_{i}, \text { если }\left(D p^{\mathrm{b}} \pm C \pi^{\mathrm{b}}\right)_{i}\left(\begin{array}{l}
\geq \\
\leq
\end{array}\right)\left(D p^{\mathrm{t}} \pm C \pi^{\mathrm{t}}+\mu^{\mathrm{t}} h^{( \pm)}\right)_{i}, \\
\left(D p^{\mathrm{t}} \pm C \pi^{\mathrm{t}}\right)_{i}+\left(\mu^{\mathrm{t}}-\mu\right) h_{i}^{( \pm)} \text {в противных случалх, } \quad i=1, \ldots, n,
\end{array}\right.
\end{gathered}
$$

где $\alpha=\mu / \mu^{\mathrm{t}}, D=\left(P^{+}\right)^{-1}, C=\operatorname{Abs}(D P), h^{( \pm)}=d \pm f, d=D B r, f=\operatorname{Abs}(D B \mathcal{R}) \rho$.

3 а м е ч а н и е 1 . Формулы для внешней оценки $\Pi^{+}=\Pi_{P^{+}}^{+}(\Pi \uplus B \mathcal{R})$ из следствия 2 вытекают также из [13, лемма 3.3]. Однако лемма 4 может быть полезна при построении не только полиэдральных оценок (как в утверждениях 1 и 4), но и других, например эллипсоидальных. 
Утверждение 2. Пусть $\mathcal{Z}=\mathcal{A} \otimes \Pi$, где $\mathcal{A}$ - интервальная матрица, $\Pi$ - это $\Pi$ политоп (1.10) с параллелепипедозначными сечениями, и пусть $P^{+}-$произвольная неособал матрица. Тогда внешняя для $\mathcal{Z}$ оченка может быть найдена по правилу $\Pi^{+}=\Pi_{P^{+}}^{+}(\mathcal{A} \otimes \Pi)$ из (3.3) :

$$
\begin{gathered}
\Pi^{+}=\Pi_{P^{+}}^{+}(\mathcal{A} \otimes \Pi)=\Pi\left(\left\{\mathcal{P}^{+\mathrm{b}}, 0\right\},\left\{\mathcal{P}^{+\mathrm{t}}, \mu^{+\mathrm{t}}\right\}\right), \mu^{+\mathrm{t}}=\mu^{\mathrm{t}}, \\
\mathcal{P}^{+\mathrm{i}}=\boldsymbol{P}_{P^{+}}^{+}\left(\mathcal{A} \circ \mathcal{P}^{\mathrm{i}}\right)=\mathcal{P}\left(P^{+}, \gamma^{+(-) \mathrm{i}}, \gamma^{+(+) \mathrm{i}}\right), \mathrm{i}=\mathrm{b}, \mathrm{t},
\end{gathered}
$$

где векторы $\gamma^{+( \pm) \mathrm{i}}, \mathrm{i}=\mathrm{b}, \mathrm{t}$, находятся с использованием любой из следующих двух формул, которые следует понимать покомпонентно:

$$
\begin{gathered}
\gamma^{+( \pm) \mathrm{i}}= \pm \max _{x \in \mathbb{E}\left(\mathcal{P}^{\mathrm{i}}\right)}\left\{ \pm\left(P^{+}\right)^{-1} \tilde{A} x+\left(\operatorname{Abs}\left(P^{+}\right)^{-1}\right) \hat{A}(\operatorname{Abs} x)\right\} \\
\gamma^{+( \pm) \mathrm{i}}= \pm \max _{A \in \mathbb{E}(\mathcal{A})}\left\{ \pm\left(P^{+}\right)^{-1} A p^{\mathrm{i}}+\left(\operatorname{Abs}\left(\left(P^{+}\right)^{-1} A P\right)\right) \pi^{\mathrm{i}}\right\}
\end{gathered}
$$

Если вдобавок $\mathcal{P}^{\mathrm{b}} \supseteq \mathcal{P}^{\mathrm{t}}$, то $\mu$-сечения $\Pi$-политопа $\Pi^{+}$будут невозрастающими.

Д о к а з а т е л ь с т в о. Формулы (3.4) вытекают из (3.2) при $l= \pm\left(\left(P^{+}\right)^{-1}\right)^{\top} \mathrm{e}^{i}$.

Ввиду следствия 1 для проверки включения $\mathcal{Z} \subseteq \Pi^{+}$достаточно установить, что при любом $\mu \in\left[0, \mu^{\mathrm{t}}\right]$ имеем $\tilde{\mathcal{P}}(\mu) \stackrel{\text { def }}{=} \boldsymbol{P}_{P^{+}}^{+}(\mathcal{A} \circ \mathcal{P}(\mu)) \subseteq \mathcal{P}^{+}(\mu)($ т.к. $\mathcal{A} \circ \mathcal{P}(\mu) \subseteq \tilde{\mathcal{P}}(\mu))$ или, с учетом представлений $\tilde{\mathcal{P}}(\mu)=\mathcal{P}\left(P^{+}, \tilde{\gamma}^{(-)}(\mu), \tilde{\gamma}^{(+)}(\mu)\right), \mathcal{P}^{+}(\mu)=\mathcal{P}\left(P^{+}, \gamma^{+(-)}(\mu), \gamma^{+(+)}(\mu)\right)$, что $\tilde{\gamma}^{(+)}(\mu) \leq$ $\gamma^{+(+)}(\mu)$ и $\tilde{\gamma}^{(-)}(\mu) \geq \gamma^{+(-)}(\mu)$ при любом $\mu \in\left[0, \mu^{\mathrm{t}}\right]$. Убедимся в выполнении первого неравенства. Для вычисления $\tilde{\gamma}^{( \pm)}(\mu)$ и $\gamma^{+( \pm)}(\mu)$ будет удобно использовать вторую из формул $(3.2)$. С учетом следствия 1 имеем $\tilde{\gamma}^{( \pm)}(\mu)= \pm \max _{A \in \mathbb{E}(\mathcal{A})}\left\{ \pm\left(P^{+}\right)^{-1} A p(\mu)+\left(\operatorname{Abs}\left(\left(P^{+}\right)^{-1} A P\right)\right) \pi(\mu)\right\}=$ $\pm \max _{A \in \mathbb{E}(\mathcal{A})}\left\{ \pm\left(P^{+}\right)^{-1} A\left(\alpha p^{\mathrm{t}}+(1-\alpha) p^{\mathrm{b}}\right)+\left(\operatorname{Abs}\left(\left(P^{+}\right)^{-1} A P\right)\right)\left(\alpha \pi^{\mathrm{t}}+(1-\alpha) \pi^{\mathrm{b}}\right)\right\}, \alpha=\mu / \mu^{\mathrm{t}}$. Bводя обозначения $f^{\mathrm{i}}(A)=\left(P^{+}\right)^{-1} A p^{\mathrm{i}}+\left(\operatorname{Abs}\left(\left(P^{+}\right)^{-1} A P\right)\right) \pi^{\mathrm{i}}$, i $=\mathrm{b}, \mathrm{t}$, и используя очевидные соотношения для функций типа $\max _{x \in \mathcal{X}}\left(f^{1}(x)+f^{2}(x)\right) \leq \max _{x \in \mathcal{X}} f^{1}(x)+\max _{x \in \mathcal{X}} f^{2}(x)$; $\max _{x \in \mathcal{X}}\left(\alpha f^{1}(x)\right)=\alpha \max _{x \in \mathcal{X}} f^{1}(x), \forall \alpha \geq 0$, а также неравенства $0 \leq \alpha=\mu / \mu^{\mathrm{t}} \leq 1$ при $\mu \in\left[0, \mu^{\mathrm{t}}\right]$, получаем $\tilde{\gamma}^{(+)}(\mu)=\max _{A \in \mathbb{E}(\mathcal{A})}\left\{\alpha f^{\mathrm{t}}(A)+(1-\alpha) f^{\mathrm{b}}(A)\right\} \leq \alpha \max _{A \in \mathbb{E}(\mathcal{A})} f^{\mathrm{t}}(A)+(1-$ $\alpha) \max _{A \in \mathbb{E}(\mathcal{A})} f^{\mathrm{b}}(A)$. Но в силу следствия 1 и второй из формул (3.4) правая часть последнего неравенства совпадает с $\gamma^{+(+)}(\mu)$. Неравенство $\tilde{\gamma}^{(+)}(\mu) \leq \gamma^{+(+)}(\mu)$ доказано. Второе неравенство $\tilde{\gamma}^{(-)}(\mu) \geq \gamma^{+(-)}(\mu)$ проверяется аналогично.

Невозрастание сечений $\Pi^{+}$следует из второй части леммы 3 (в которой взято $\mathcal{Z}=\Pi^{+}$) ввиду включения $\mathcal{P}^{+\mathrm{b}} \supseteq \mathcal{P}^{+\mathrm{t}}$, вытекающего из леммы 1 и определения касающейся оценки.

3 а м е ч а н и е 2 . Как видно из доказательства утверждения 2 , внешние оценки $\Pi_{P^{+}}^{+}(\mathcal{A} \otimes \Pi)$ не обязаны быть касающимися для $\mathcal{A} \otimes \Pi$ (в отличие от $\boldsymbol{P}_{P^{+}}^{+}(\mathcal{A} \circ \mathcal{P})$ и $\Pi_{P^{+}}^{+}(\Pi \uplus \mathcal{R})$ ).

Рассмотрим способы построения внутренних оценок и опять начнем с оценок в $\mathbb{R}^{n}$.

Введем множество матриц $\mathcal{G}^{r \times n}=\left\{\Gamma=\left\{\gamma_{\alpha}^{\beta}\right\} \in \mathbb{R}^{r \times n} \mid\|\Gamma\| \leq 1\right\}$, где $\|\Gamma\|=\max _{1 \leq \alpha \leq r} \sum_{\beta=1}^{n}\left|\gamma_{\alpha}^{\beta}\right|$.

Пусть $\mathcal{P}^{k}=\mathcal{P}\left[p^{k}, \bar{P}^{k}\right], k=1,2, \bar{P}^{1} \in \mathbb{R}^{n \times n}, \bar{P}^{2} \in \mathbb{R}^{n \times r}$. Внутренние параллелотопозначные оценки для $\mathcal{Q}=\mathcal{P}^{1}+\mathcal{P}^{2}$ могут быть найдены в виде $\boldsymbol{P}_{\Gamma^{1}, \Gamma^{2}}^{-}(\mathcal{Q}) \stackrel{\text { def }}{=} \mathcal{P}\left[p^{1}+p^{2}, \bar{P}^{1} \Gamma^{1}+\bar{P}^{2} \Gamma^{2}\right]$, где $\Gamma^{1}, \Gamma^{2}-$ произвольные матрицы, удовлетворяющие условиям $\Gamma^{1} \in \mathcal{G}^{n \times n}, \Gamma^{2} \in \mathcal{G}^{r \times n}$ (см. лемму 3.1 в ссылке [14] из работы [13]).

Семейство внутренних для $\mathcal{Q}=\mathcal{A} \circ \mathcal{P}$ оценок, включающее введенные ранее в [18;21], дает следующее утверждение.

Утверждение 3. Пусть $\mathcal{P}=\mathcal{P}[p, \bar{P}]$, где $\bar{P} \in \mathbb{R}^{n \times n}, u \mathcal{A}$-интервальная матрица. Пусть $\Gamma^{1}, \Gamma^{2}$ - произвольные матрицы, удовлетворяющие $\Gamma^{1}, \Gamma^{2} \in \mathcal{G}^{n \times n}, J=\left\{j_{1}, \ldots, j_{n}\right\}-$ произвольная перестановка чисел $\{1, \ldots, n\}$, а параллелотоп $\mathcal{P}^{-}$определяется формулами

$$
\begin{gathered}
\mathcal{P}^{-}=\boldsymbol{P}_{J, \Gamma^{1}, \Gamma^{2}}^{-}(\mathcal{A} \circ \mathcal{P}) \stackrel{\text { def }}{=} \mathcal{P}\left[\tilde{A} p, \tilde{A} \bar{P} \Gamma^{1}+(\operatorname{diag} \nu) \Gamma^{2}\right], \\
\nu_{i}=\hat{a}_{i}^{j_{i}} \eta_{j_{i}}, \quad i=1, \ldots, n, \quad \eta=\max \left\{0, \operatorname{Abs} p-\operatorname{Abs}\left(\bar{P} \Gamma^{1}\right) \mathrm{e}\right\} .
\end{gathered}
$$


Тогда $\mathcal{P}^{-} \subseteq \mathcal{A} \circ \mathcal{P}$. Eсли $\operatorname{det} \tilde{A} \neq 0, \operatorname{det} \bar{P} \neq 0, a \Gamma^{1} u \Gamma^{2}$ взяты в виде $\Gamma^{1}=\Gamma, \Gamma^{2}=(\operatorname{diag} \beta) \tilde{A} \bar{P} \Gamma$, где $\beta_{i}=\left(\mathrm{e}^{i^{\top}} \operatorname{Abs}(\tilde{A} \bar{P} \Gamma) \mathrm{e}\right)^{-1}, i=1, \ldots, n$, а матрица $\Gamma \in \mathcal{G}^{n \times n}$ является неособой $(\operatorname{det} \Gamma \neq 0)$, то $\mathcal{P}^{-}$оказывается невырожденным параллелелепипедом, совпадающим с $[18,(5)]$.

Д о к а з а т е л ь с т в о проводится по схеме из [21, утверждение 1] и [18, теорема 1] с необходимыми модификациями.

Несложно привести пример того, что введение независимого параметра $\Gamma^{2}$ позволяет при некоторых предположениях построить невырожденный $\mathcal{P}^{-}$вида (3.5) при вырожденном $\mathcal{P}$.

Внутренние элементарные оценки для множеств в $\mathbb{R}^{n+1}$ будем строить в виде $\Pi$-цилиндров.

Утверждение 4. Пусть $\Pi=\Pi\left(\left\{\mathcal{P}^{\mathrm{b}}, 0\right\},\left\{\mathcal{P}^{\mathrm{t}}, \mu^{\mathrm{t}}\right\}\right)-$ это $\Pi$-цилиндр с одинаковыми параллелотопозначными сечениями $\mathcal{P}^{\mathrm{b}}=\mathcal{P}^{\mathrm{t}}=\mathcal{P}\left[p^{\mathrm{t}}, \bar{P}^{\mathrm{t}}\right]$, a $\mathcal{P}=\mathcal{P}[p, \bar{P}]-$ nараллелотоп с $\bar{P} \in$ $\mathbb{R}^{n \times r}$, такой что $0 \in \mathcal{P}$. Тогда внутренней оценкой для $\Pi \uplus \mathcal{P}$ слуюит любой $\Pi$-цилиндр вида

$$
\begin{gathered}
\Pi^{-}=\Pi_{h, \Gamma^{1}, \Gamma^{2}}^{-}(\Pi \uplus \mathcal{P}) \stackrel{\text { def }}{=} \Pi\left(\left\{\mathcal{P}^{-\mathrm{b}}, 0\right\},\left\{\mathcal{P}^{-\mathrm{t}}, \mu^{-\mathrm{t}}\right\}\right), \\
\mu^{-\mathrm{t}}=\mu^{\mathrm{t}}-h, \mathcal{P}^{-\mathrm{b}}=\mathcal{P}^{-\mathrm{t}}=\boldsymbol{P}_{\Gamma^{1}, \Gamma^{2}}^{-}\left(\mathcal{P}^{\mathrm{t}}+h \mathcal{P}\right)=\mathcal{P}\left[p^{\mathrm{t}}+h p, \bar{P}^{\mathrm{t}} \Gamma^{1}+h \bar{P} \Gamma^{2}\right],
\end{gathered}
$$

соответствуюший допустимым значениям параметров $h \in\left[0, \mu^{\mathrm{t}}\right], \Gamma^{1} \in \mathcal{G}^{n \times n} u \Gamma^{2} \in \mathcal{G}^{r \times n}$.

Д о к а з а т е л ь с т в о. Применяя лемму 4 к $\Pi$-цилиндру $\Pi=\bigcup_{0 \leq \mu \leq \mu^{\mathrm{t}}}\{\mathcal{P}(\mu), \mu\}$ с $\mathcal{P}^{\mathrm{b}}=$ $\mathcal{P}^{\mathrm{t}}$, получаем следующие равенства и включения для $\mu$-сечений $\hat{\mathcal{X}}(\mu)$ множества $\Pi \uplus \mathcal{P}=\hat{\mathcal{Z}}$ : $\hat{\mathcal{X}}(\mu)=\operatorname{co}\left(\mathcal{P}(\mu) \cup\left(\mathcal{P}\left(\mu^{\mathrm{t}}\right)+\left(\mu^{\mathrm{t}}-\mu\right) \mathcal{P}\right)\right)=\operatorname{co}\left(\mathcal{P}^{\mathrm{t}} \cup\left(\mathcal{P}^{\mathrm{t}}+\left(\mu^{\mathrm{t}}-\mu\right) \mathcal{P}\right)\right)=\mathcal{P}^{\mathrm{t}}+\left(\mu^{\mathrm{t}}-\mu\right) \mathcal{P} \supseteq \mathcal{P}^{\mathrm{t}}+h \mathcal{P} \supseteq$ $\mathcal{P}\left[p^{\mathrm{t}}+h p, \bar{P}^{\mathrm{t}} \Gamma^{1}+h \bar{P} \Gamma^{2}\right]$. Здесь были последовательно учтены условия $\mathcal{P}^{\mathrm{b}}=\mathcal{P}^{\mathrm{t}}, 0 \in \mathcal{P}, \mu \leq \mu^{\mathrm{t}}-h$ и использована указанная выше внутренняя оценка для $\mathcal{P}^{\mathrm{t}}+h \mathcal{P}$.

Утверждение 5. Пусть $\Pi=\Pi\left(\left\{\mathcal{P}^{\mathrm{b}}, 0\right\},\left\{\mathcal{P}^{\mathrm{t}}, \mu^{\mathrm{t}}\right\}\right)-$ это $\Pi$-иилиндр с $\mathcal{P}^{\mathrm{b}}=\mathcal{P}^{\mathrm{t}}$, a $\mathcal{A}-$ интервальная матрица. Тогда внутренней оченкой для $\mathcal{A} \otimes \Pi$ служит любой П-цилиндр

$$
\Pi^{-}=\Pi_{J, \Gamma^{1}, \Gamma^{2}}^{-}(\mathcal{A} \otimes \Pi) \stackrel{\text { def }}{=} \Pi\left(\left\{\mathcal{P}^{-\mathrm{b}}, 0\right\},\left\{\mathcal{P}^{-\mathrm{t}}, \mu^{-\mathrm{t}}\right\}\right), \mu^{-\mathrm{t}}=\mu^{\mathrm{t}}, \mathcal{P}^{-\mathrm{b}}=\mathcal{P}^{-\mathrm{t}}=\boldsymbol{P}_{J, \Gamma^{1}, \Gamma^{2}}^{-}\left(\mathcal{A} \circ \mathcal{P}^{\mathrm{t}}\right),
$$

где фигурирует внутренняя оценка для $\mathcal{A} \circ \mathcal{P}^{\mathrm{t}}$ из утверждения 3 , а $J и \Gamma^{1}, \Gamma^{2} \in \mathcal{G}^{n \times n}-$ произвольные значения описанных там допустимых параметров.

Д о к а з а т е л ь с т в о следует из утверждения 3 и равенства всех $\mu$-сечений $\Pi$.

3 а м е ч а н и е 3 . Оценки (3.5) обладают тем свойством, что в случае $\mathcal{P} \ni 0$ получается $\nu=\eta=0$, т. е. фактически не используется то, что матрица $\mathcal{A}$ - интервальная. В таком случае может быть полезнее использовать так называемые простые оценки $\boldsymbol{P}_{A}^{-}(\mathcal{A} \circ \mathcal{P})$ из [18] вида $\boldsymbol{P}_{A}^{-}(\mathcal{A} \circ \mathcal{P})=A \mathcal{P}=\mathcal{P}[A p, A \bar{P}]$, где $A \in \mathcal{A}$, и вместо (3.7) строить внутренние оценки для $\mathcal{A} \otimes \Pi$ в виде $\Pi$-цилиндров вида

$\Pi^{-}=\Pi_{A}^{-}(\mathcal{A} \otimes \Pi) \stackrel{\text { def }}{=} \Pi\left(\left\{\mathcal{P}^{-\mathrm{b}}, 0\right\},\left\{\mathcal{P}^{-\mathrm{t}}, \mu^{-\mathrm{t}}\right\}\right), \mu^{-\mathrm{t}}=\mu^{\mathrm{t}}, \mathcal{P}^{-\mathrm{b}}=\mathcal{P}^{-\mathrm{t}}=\boldsymbol{P}_{A}^{-}\left(\mathcal{A} \circ \mathcal{P}^{\mathrm{t}}\right)=\mathcal{P}\left[A p^{\mathrm{t}}, A \bar{P}^{\mathrm{t}}\right]$,

где параметром служит $A \in \mathcal{A}$. Напомним, что максимальные по объему простые оценки $\boldsymbol{P}_{A}^{-}(\mathcal{A} \circ \mathcal{P})$ могут быть найдены среди оценок, соответствующих вершинам интервальной матрицы $\mathcal{A}$, а именно $\max _{A \in \mathcal{A}} \operatorname{vol}(A \mathcal{P})=\max _{A \in \mathbb{E}(\mathcal{A})} \operatorname{vol}(A \mathcal{P})=\max _{A \in \mathbb{E}(\mathcal{A})}|\operatorname{det} A| \cdot \operatorname{vol} \mathcal{P}[18]$.

\section{4. Полиэдральные оценки множеств достижимости $\mathcal{Z}[k]$ и $\mathcal{X}[k]$}

Базируясь на введенных элементарных оценках, опишем способы построения полиэдральных оценок множеств достижимости. Начнем с внешних оценок, предложенных в [19]. 
Теорема 2. Пусть $\mathcal{Z}[k]$ - множества достижимости системы (1.1), (1.3)-(1.8) и въполнено предположение 1 . Пусть П -политопь $\Pi^{+}[k]$ находятся из следующих соотношений:

$$
\begin{aligned}
& \Pi^{1+}[k]=\Pi_{P^{+}[k]}^{+}\left(\mathcal{A}[k] \otimes \Pi^{+}[k-1]\right) \oplus v[k], \quad k=1, \ldots, N ; \quad \Pi^{+}[0]=\mathcal{P}_{0} \times\left[0, \mu_{0}\right] ; \\
& \Pi^{+}[k]=\Pi_{P^{+}[k]}^{+}\left(\Pi^{1+}[k] \uplus B[k] \mathcal{R}[k]\right), \quad k=1, \ldots, N,
\end{aligned}
$$

которые конкретизируются с помощъю формул из утверждения 2 и следствия 2. Тогда политопы $\Pi^{+}[k]$ являются внешними оченками для $\mathcal{Z}[k]\left(\mathcal{Z}[k] \subseteq \Pi^{+}[k], k=1, \ldots, N\right)$ при любых неособых матрицах ориентации $P^{+}[k] \in \mathbb{R}^{n \times n}, k=1, \ldots, N$. При этом сечения политопов $\Pi^{+}[k]$ и $\Pi^{1+}[k]$ не возрастают.

Д о к а з а т е л ь с т в о. Справедливость теоремы вытекает из теоремы 1 , утверждений 1 и 2 и монотонности по включению задействованных операций с множествами.

Следствие 3. Пусть $\mathcal{X}[k]$ - множества достижимости системы (1.1)-(1.4) и выполнено предположение 1 . Пусть параллелепипеды $\mathcal{P}^{+}[k]$ находятся из следующих соотношений:

$$
\begin{gathered}
\mathcal{P}^{0+}[k]=\boldsymbol{P}_{P^{+}[k]}^{+}\left(\mathcal{A}[k] \circ \mathcal{P}^{0+}[k-1]\right)+v[k], \quad k=1, \ldots, N ; \quad \mathcal{P}^{0+}[0]=\mathcal{P}_{0} ; \\
\mathcal{P}^{+}[k]=\boldsymbol{P}_{P^{+}[k]}^{+}\left(\left(\mathcal{A}[k] \circ \mathcal{P}^{+}[k-1]+v[k]\right) \cup\left(\mathcal{P}^{0+}[k]+\mu_{0} B[k] \mathcal{R}[k]\right)\right) \\
=\boldsymbol{P}_{P^{+}[k]}^{+}\left(\boldsymbol{P}_{P^{+}[k]}^{+}\left(\mathcal{A}[k] \circ \mathcal{P}^{+}[k-1]+v[k]\right) \cup \boldsymbol{P}_{P^{+}[k]}^{+}\left(\mathcal{P}^{0+}[k]+\mu_{0} B[k] \mathcal{R}[k]\right)\right), \\
k=1, \ldots, N ; \quad \mathcal{P}^{+}[0]=\mathcal{P}_{0} .
\end{gathered}
$$

Тогда они являются внешними оценками для $\mathcal{X}[k]: \mathcal{X}[k] \subseteq \mathcal{P}^{+}[k], k=1, \ldots, N$, каковы бъ ни были неособые матрицы ориентации $P^{+}[k], k=1, \ldots, N$.

Д о к а з а т е л ь с т в о. Включения $\mathcal{X}[k] \subseteq \mathcal{P}^{+}[k]$ следуют из теорем 1 и 2 , поскольку соотношения (4.2) фактически описывают динамику верхних и нижних сечений политопов из (4.1): $\mathcal{P}^{0+}[k]=\mathcal{P}^{+\mathrm{t}}[k], \mathcal{P}^{+}[k]=\mathcal{P}^{+\mathrm{b}}[k]$. При этом второе равенство в выражении для $\mathcal{P}^{+}[k]$ в (4.2) вытекает из равенств типа $\boldsymbol{P}_{P^{+}}^{+}\left(\boldsymbol{P}_{P^{+}}^{+}\left(\mathcal{X}^{1}\right) \cup \mathcal{X}^{2}\right)=\boldsymbol{P}_{P^{+}}^{+}\left(\mathcal{X}^{1} \cup \mathcal{X}^{2}\right)$, которые проверяются с помощью аппарата опорных функций.

3 а м е ч а н и е 4. Соотношения (4.1) и (4.2) описывают параметризованные семейства полиэдральных трубок в $\mathbb{R}^{n+1}$ и $\mathbb{R}^{n}$ соответственно, где параметром служит последовательность матриц ориентации $P^{+}[\cdot]$. При выполнении предположения 2 матрицы $P^{+}[k]$ могут быть найдены по аналогии с [13] из соотношений $P^{+}[k]=\tilde{A}[k] P^{+}[k-1], k=1, \ldots, N ; P^{+}[0]=P$, где $P$ - произвольная неособая матрица; теперь она будет параметром семейства (более узкого). При этом в случае $\hat{A}[j] \equiv 0$ оценки из (4.1) совпадут с оценками из [13, теорема 4.2$]$ и будут касающимися для МД $\mathcal{Z}[k]$, а параллелепипеды $\mathcal{P}^{+}[k]$ из (4.2) будут касающимися оценками для МД $\mathcal{X}[k]$. Выбор постоянных матриц ориентации $P^{+}[k] \equiv P$ может привести к гораздо более грубым оценкам ввиду известного в интервальном анализе "эффекта обертывания".

Обратимся теперь к построению внутренних оценок.

Введем семейство трубок $\Pi^{-}[\cdot]$, удовлетворяющих соотношениям

$$
\begin{array}{r}
\Pi^{1-}[k]=\Pi_{J[k], \Gamma^{1}[k], \Gamma^{2}[k]}^{-}\left(\mathcal{A}[k] \otimes \Pi^{-}[k-1]\right) \oplus v[k], \quad k=1, \ldots, N ; \\
\Pi^{-}[k]=\Pi_{h[k], \Gamma^{3}[k], \Gamma^{4}[k]}^{-}\left(\Pi^{1-}[k] \uplus B[k] \mathcal{R}[k]\right), \quad k=1, \ldots, N ; \\
\Pi^{-}[0]=\mathcal{P}_{0} \times\left[0, \mu_{0}\right]=\Pi\left(\left\{\mathcal{P}_{0}, 0\right\},\left\{\mathcal{P}_{0}, \mu_{0}\right\}\right),
\end{array}
$$

где параметры $J[\cdot], \Gamma^{1}[\cdot], \Gamma^{2}[\cdot], h[\cdot], \Gamma^{3}[\cdot], \Gamma^{4}[\cdot]$ отвечают условиям

$$
h[j] \geq 0, j=1, \ldots, N, \sum_{j=1}^{N} h[j] \leq \mu_{0} ; \quad \Gamma^{1}[j], \Gamma^{2}[j], \Gamma^{3}[j] \in \mathcal{G}^{n \times n}, \Gamma^{4}[j] \in \mathcal{G}^{n_{u} \times n}, j=1, \ldots, N,
$$

$J[j]$ - произвольные перестановки чисел $\{1, \ldots, n\}$; называем такие значения допустимыми. 
Теорема 3. Пусть $\mathcal{Z}[k]$ - множества достижимости системы (1.1), (1.3)-(1.8) и выполнено предположсене 1. Пусть П-иилиндры $\Pi^{-}[k]$ находятся из соотношений (4.3), которые конкретизируются с помощъю формул (3.7), (3.5), (3.6). Тогда $\Pi^{-}[k]$ являются внутренними оиенками для $\mathcal{Z}[k]$ при произвольных допустимых значениях параметров $J[\cdot], \Gamma^{1}[\cdot], \Gamma^{2}[\cdot], h[\cdot], \Gamma^{3}[\cdot], \Gamma^{4}[\cdot]: \quad \Pi^{-}[k] \subseteq \mathcal{Z}[k], k=1, \ldots, N$.

Д о к а з а т е л ь с т в о аналогично доказательству теоремы 2 с опорой на утверждения 4 и 5 . Выполнение требуемого в утверждении 4 условия $0 \in \mathcal{R}[k]$ следует из (1.9). Условия на $h[\cdot]$ из (4.4) обеспечивают ввиду (3.6), что $\mu^{-\mathrm{t}}[k] \geq 0, k=1, \ldots, N$.

Следствие 4. Пусть $\mathcal{X}[k]$ - множества достижимости системы (1.1)-(1.4) и выполнено предположение 1 . Пусть параллелотопь $\mathcal{P}^{-}[k]$ находятся из соотношений

$$
\begin{aligned}
& \mathcal{P}^{0-}[k]=\boldsymbol{P}_{J[k], \Gamma^{1}[k], \Gamma^{2}[k]}^{-}\left(\mathcal{A}[k] \circ \mathcal{P}^{-}[k-1]\right)+v[k], k=1, \ldots, N ; \\
& \mathcal{P}^{-}[k]=\boldsymbol{P}_{\Gamma^{3}[k], \Gamma^{4}[k]}^{-}\left(\mathcal{P}^{0-}[k]+h[k] B[k] \mathcal{R}[k]\right), \quad k=1, \ldots, N ; \quad \mathcal{P}^{-}[0]=\mathcal{P}_{0},
\end{aligned}
$$

при таких же допустимых значениях параметров $J[\cdot], \Gamma^{1}[\cdot], \Gamma^{2}[\cdot], h[\cdot], \Gamma^{3}[\cdot], \Gamma^{4}[\cdot]$, как и в теореме 3. Тогда они являются внутренними оценками для $\mathcal{X}[k]: \mathcal{P}^{-}[k] \subseteq \mathcal{X}[k], k=1, \ldots, N$.

Д о к а з а т е л ь с т в о. Включения $\mathcal{P}^{-}[k] \subseteq \mathcal{X}[k]$ вытекают из теорем 1 и 3 , поскольку соотношения (4.5) фактически описывают динамику $\mu$-сечений цилиндров из (4.3): $\mathcal{P}^{-}[k]=$ $\mathcal{P}^{-\mathrm{t}}[k]=\mathcal{P}^{-\mathrm{b}}[k]$ (а $\mathcal{P}^{0-}[k]$ совпадают с $\mu$-сечениями цилиндров $\left.\Pi^{1-}[k]\right)$.

Можно дать и другое доказательство следствия 4. Действительно, несложно видеть, что если рассмотреть МД $\mathcal{X}[k ; h[\cdot]]$ системы $(1.1),(1.3),(1.4)$ с $x[0] \in \mathcal{X}_{0}$ и ограничениями на $u[\cdot]$ вида $u[j] \subseteq h[j] \mathcal{R}[j], j=1, \ldots, N$, то при условиях на $h[\cdot]$ из (4.4) будем иметь $\mathcal{X}[k ; h[\cdot]] \subseteq \mathcal{X}[k]$, $k=1, \ldots, N$. Поэтому далее достаточно использовать конструкции для построения внутренних оценок МД $\mathcal{X}[k ; h[\cdot]]$ систем с геометрическими ограничениями и элементарные внутренние оценки в $\mathbb{R}^{n}$, описанные в разд. 3 .

3 а м е ч а н и е 5 . Соотношения (4.3) и (4.5) описывают параметризованные семейства полиэдральных трубок в $\mathbb{R}^{n+1}$ и $\mathbb{R}^{n}$ соответственно, где параметрами служат функции $h[\cdot]$, $J[\cdot], \Gamma^{i}[\cdot], i=1,2,3,4$. Если при каких-то $k \in\{1, \ldots, N\}$ оказывается, что $\mathcal{P}^{-\mathrm{t}}[k] \ni 0$, то ввиду замечания 3 может быть полезно при таких $k$ вместо формул для $\Pi^{1-}[k]$ из $(4.3)$ использовать формулы

$$
\Pi^{1-}[k]=\Pi_{A[k]}^{-}\left(\mathcal{A}[k] \otimes \Pi^{-}[k-1]\right) \oplus v[k]
$$

типа (3.8) и, аналогично, вместо формул для $\mathcal{P}^{0-}[k]$ из (4.5) использовать формулы

$$
\mathcal{P}^{0-}[k]=\boldsymbol{P}_{A[k]}^{-}\left(\mathcal{A}[k] \circ \mathcal{P}^{-}[k-1]\right)+v[k],
$$

где матрицы $A[k] \in \mathcal{A}[k]$ играют роль параметра оценки.

\section{Заключение}

Исследована задача достижимости для многошаговых систем с исходно линейной структурой и неопределенностями в начальных условиях, матрицах и аддитивных воздействиях, причем последние стеснены интегральными ограничениями. Представлены соотношения, обеспечивающие точное описание множеств достижимости, и предложены способы построения параметризованных семейств внешних и внутренних полиэдральных оценок для них. При этом рассматриваются не только МД $\mathcal{X}[k]$ в исходном пространстве, но и МД $\mathcal{Z}[k]$ в "расширенном" пространстве, обладающие важным полугрупповым свойством. Для множеств $\mathcal{Z}[k]$ строятся внешние и внутренние оценки в виде политопов специального типа, которые называем П-политопами и $\Pi$-иилиндрами. Определенные сечения таких оценок дают внешние параллелепипедозначные и внутренние параллелотопозначные оценки для $\mathcal{X}[k]$. Хотя такие оценки 
могут оказаться несколько грубыми, их можно легко вычислить по явным формулам из систем рекуррентных соотношений, и они могут быть довольно информативными, в то время как точное построение МД достаточно затруднительно. Введение семейств оценок позволяет более точно оценить МД в виде пересечения нескольких внешних оценок и объединения нескольких внутренних. В [19] приведен пример построения внешних оценок для двумерной системы. Примеры численного построения описываемых в статье двусторонних оценок с использованием компьютерной графики для того случая и для систем большей размерности будут представлены в отдельной публикации. Отметим также, что ввиду полугруппового свойства предложенные оценки для $\mathcal{Z}[k]$ могут быть модифицированы с целью получения оценок для МД систем с фазовыми ограничениями и для информационных множеств $[2 ; 3 ; 7]$.

\section{СПИСОК ЛИТЕРАТУРЫ}

1. Красовский Н.Н. Теория управления движением. М.: Наука, 1968. 476 с.

2. Куржанский А.Б. Управление и наблюдение в условиях неопределенности. М.: Наука, 1977. $392 \mathrm{c}$.

3. Kurzhanski A.B., Varaiya P. Dynamics and control of trajectory tubes: theory and computation. Basel: Birkhäuser, 2014. 445 p. (Systems \& Control: Foundations \& Applications, Book 85). doi: 10.1007/978-3-319-10277-1.

4. Kurzhanski A.B., Daryin A.N. Dynamic programming for impulse feedback and fast controls: The linear systems case. London: Springer, 2020. 275p. (LNCIS, vol. 468.) doi: 10.1007/978-1-4471-7437-0 .

5. Лотов А.В. Метод построения внешней полиэдральной оценки трубки траекторий нелинейной динамической системы // Докл. АН. 2017. Т. 472, №1. С. 18-22.

6. Guseinov K.G., Ozer O., Akyar E., Ushakov V.N. The approximation of reachable sets of control systems with integral constraint on controls // Nonlinear Differential Equations and Appl. 2007. Vol. 14, iss. 1-2. P. 57-73. doi: 10.1007/s00030-006-4036-6.

7. Kurzhanski A.B., Vályi I. Ellipsoidal calculus for estimation and control. Boston: Birkhäuser, 1997. $321 \mathrm{p}$.

8. Черноусько Ф.Л. Оценивание фазового состояния динамических систем. Метод эллипсоидов. М.: Наука, 1988. 319 с.

9. Ананьев Б.И., Гусев М.И., Филиппова Т.Ф. Управление и оценивание состояний динамических систем с неопределенностью. Новосибирск: Изд-во СО РАН, 2018. 193 с.

10. Le V.T.H., Stoica C., Alamo T., Camacho E.F., Dumur D. Zonotopes: From guaranteed stateestimation to control. Croydon: Wiley-ISTE, 2013. 150 p. doi: 10.1002/9781118761588.

11. Sharma U., Thangavel S., Gottu Mukkula A.R., Paulen R. Effective recursive parallelotopic bounding for robust output-feedback control // IFAC-PapersOnLine. 2018. Vol. 51, iss. 15. P. 1032-1037. doi: 10.1016/j.ifacol.2018.09.058 .

12. Dreossi T., Dang T., Piazza C. Reachability computation for polynomial dynamical systems // Formal Methods in System Design. 2017. Vol. 50, iss. 1. P. 1-38. doi: 10.1007/s10703-016-0266-3 .

13. Костоусова Е.K. О внешнем полиэдральном оценивании множеств достижимости в "расширенном" пространстве для линейных многошаговых систем с интегральными ограничениями на управление // Вычисл. технологии. 2004. Т. 9, № 5. С. 54-72.

14. Tang W., Wang Z., Shen Y. Interval Estimation methods for discrete-time linear time-invariant systems // Systems \& Control Letters. 2019. Vol. 123. P. 69--74. doi: 10.1016/j.sysconle.2018.11.001.

15. Filippova T.F., Matviychuk O.G. Estimates of reachable sets of control systems with bilinearquadratic nonlinearities // Ural Math. J. 2015. Vol. 1, no. 1. P. 45-54. doi: 10.15826/umj.2015.1.004.

16. Mazurenko S.S. Partial differential equation for evolution of star-shaped reachability domains of differential inclusions // Set-Valued Var. Anal. 2016. Vol. 24, iss. 2. P. 333-354. doi: 10.1007/s11228015-0345-4.

17. Синяков В.В. Метод вычисления внешних и внутренних аппроксимаций множеств достижимости билинейных дифференциальных систем // Дифференц. уравнения. 2015. Т. 51, № 8. С. 1101-1114.

18. Костоусова E.K. О полиэдральных оценках множеств достижимости многошаговых систем с билинейной неопределенностью // Автоматика и телемеханика. 2011. № 9. С. 49-60.

19. Kostousova E.K. State estimates of bilinear discrete-time systems with integral constraints through polyhedral techniques // IFAC-PapersOnLine. 2018. Vol. 51, iss. 32. P. 245-250. doi: 10.1016/j.ifacol.2018.11.389. 
20. Chernousko F.L., Rokityanskii D.Ya. Ellipsoidal bounds on reachable sets of dynamical systems with matrices subjected to uncertain perturbations // J. Optim. Theory Appl. 2000. Vol. 104, iss. 1. P. 1-19. doi: 10.1023/A:1004687620019.

21. Костоусова Е.К. О полиэдральных оценках множеств достижимости дифференциальных систем с билинейной неопределенностью // Тр. Ин-та математики и механики УрО РАН. 2012. Т. 18 , № 4. C. $195-210$.

Поступила 13.11.2019

После доработки 22.01.2020

Костоусова Елена Кирилловна

д-р физ.-мат. наук

ведущий науч. сотрудник

Институт математики и механики им. Н. Н. Красовского УрО РАН

г. Екатеринбург

e-mail: kek@imm.uran.ru

\section{REFERENCES}

1. Krasovskii N.N. Teoriya upravleniya dvizheniem [Theory of motion control]. Moscow: Nauka Publ., 1968, $476 \mathrm{p}$.

2. Kurzhanski A.B. Upravlenie i nablyudenie v usloviyakh neopredelennosti [Control and observation under the conditions of uncertainty]. Moscow: Nauka Publ., 1977, 392 p.

3. Kurzhanski A.B., Varaiya P. Dynamics and control of trajectory tubes: theory and computation. Ser. Systems \& Control: Foundations \& Applications, Book 85, Basel: Birkhäuser, 2014, 445 p. doi: 10.1007/978-3-319-10277-1 .

4. Kurzhanski A.B., Daryin A.N. Dynamic programming for impulse feedback and fast controls: The linear systems case. LNCIS, vol. 468, London: Springer, 2020, 275 p. doi: 10.1007/978-1-4471-7437-0 .

5. Lotov A.V. Method for constructing an external polyhedral estimate of the trajectory tube for a nonlinear dynamic system. Dokl. Math., 2017, vol. 95, no. 1, pp. 95-98. doi: 10.1134/S1064562417010045.

6. Guseinov K.G., Ozer O., Akyar E., Ushakov V.N. The approximation of reachable sets of control systems with integral constraint on controls. Nonlinear Differential Equations and Appl., 2007, vol. 14, no. 1-2, pp. 57-73. doi: 10.1007/s00030-006-4036-6.

7. Kurzhanski A.B., Vályi I. Ellipsoidal calculus for estimation and control. Boston: Birkhäuser, 1997, 321 p. ISBN: 978-0-8176-3699-9.

8. Chernousko F.L. State estimation for dynamic systems. Boca Raton: CRC Press, 1994, 304 p. ISBN: 0-8493-4458-1. Original Russian text published in Chernous'ko F.L. Otsenivanie fazovogo sostoyaniya dinamicheskikh sistem. Metod ellipsoidov. Moscow: Nauka Publ., 1988, 319 p.

9. Ananyev B.I., Gusev M.I., Filippova T.F. Upravlenie $i$ otsenivanie sostoyanii dinamicheskikh sistem $s$ neopredelennost'yu [Control and estimation of states of dynamic systems with uncertainty]. Novosibirsk: Izdatelstvo SO RAN, 2018, 193 p. ISBN: 978-5-7692-1624-4.

10. Le V.T.H., Stoica C., Alamo T., Camacho E.F., Dumur D. Zonotopes: From guaranteed state-estimation to control. Croydon: Wiley-ISTE, 2013. 150 p. doi: 10.1002/9781118761588.

11. Sharma U., Thangavel S., Gottu Mukkula A.R., Paulen R. Effective recursive parallelotopic bounding for robust output-feedback control. IFAC-PapersOnLine, 2018, vol. 51, no. 15, pp. 1032-1037. doi: 10.1016/j.ifacol.2018.09.058.

12. Dreossi T., Dang T., Piazza C. Reachability computation for polynomial dynamical systems. Formal Methods in System Design, 2017, vol. 50, no. 1, pp. 1-38. doi: 10.1007/s10703-016-0266-3 .

13. Kostousova E.K. Outer polyhedral estimates of reachable sets in the "extended" phase space for linear discrete systems with integral bounds on controls. Vychisl. Tekhnol., 2004, vol. 9, no. 5, pp. 54-72 (in Russian).

14. Tang W., Wang Z., Shen Y. Interval Estimation methods for discrete-time linear time-invariant systems. Systems and Control Letters, 2019, vol. 123, pp. 69-74. doi: 10.1016/j.sysconle.2018.11.001.

15. Filippova T.F., Matviychuk O.G. Estimates of reachable sets of control systems with bilinear-quadratic nonlinearities. Ural Math. J., 2015, vol. 1, no. 1, pp. 45-54. doi: 10.15826/umj.2015.1.004. 
16. Mazurenko S.S. Partial differential equation for evolution of star-shaped reachability domains of differential inclusions. Set-Valued Var. Anal., 2016, vol. 24, no. 2, pp. 333-354.

doi: $10.1007 / \mathrm{s} 11228-015-0345-4$.

17. Sinyakov V.V. Method for computing exterior and interior approximations to the reachability sets of bilinear differential systems. Differential Equations. 2015, vol. 51, no. 8, pp. 1097-1111. doi: $10.1134 /$ S0012266115080145 .

18. Kostousova E.K. On polyhedral estimates for reachable sets of discrete-time systems with bilinear uncertainty. Automation and Remote Control, 2011, vol. 72, no. 9, pp. 1841-1851. doi: $10.1134 /$ S0005117911090062 .

19. Kostousova E.K. State estimates of bilinear discrete-time systems with integral constraints through polyhedral techniques. IFAC-PapersOnLine, 2018, vol. 51, no. 32, pp. 245-250. doi: $10.1016 /$ j.ifacol.2018.11.389 .

20. Chernousko F.L., Rokityanskii D.Ya. Ellipsoidal bounds on reachable sets of dynamical systems with matrices subjected to uncertain perturbations. J. Optimiz. Theory Appl., 2000, vol. 104, no. 1, pp. 1-19. doi: 10.1023/A:1004687620019.

21. Kostousova E.K. On polyhedral estimates for reachable sets of differential systems with bilinear uncertainty. Trudy Instituta Matematiki i Mekhaniki UrO RAN, 2012, vol. 18, no. 4, pp. 195-210 (in Russian).

Received November 13, 2019

Revised January 22, 2020

Accepted January 27, 2020

Elena Kirillovna Kostousova, Dr. Phys.-Math. Sci., Krasovskii Institute of Mathematics and Mechanics of the Ural Branch of the Russian Academy of Sciences, Yekaterinburg, 620108 Russia, e-mail: kek@imm.uran.ru .

Cite this article as: E. K. Kostousova. On polyhedral estimation of reachable sets in the "extended" space for discrete-time systems with uncertain matrices and integral constraints, Trudy Instituta Matematiki i Mekhaniki URO RAN, 2020, vol. 26, no. 1, pp. 141-155. 